\title{
On the First-Order Approach in Principal-Agent Models with Hidden Borrowing and Lending*
}

\author{
Árpád Ábrahám, ${ }^{\dagger}$ Sebastian Koehne, ${ }^{\ddagger}$ and Nicola Pavoni ${ }^{\S}$
}

February 2010

\begin{abstract}
Moral hazard models with hidden asset markets are useful to study several interesting problems such as unemployment insurance, income taxation, executive compensation, or human capital policies. Without the use the first-order condition approach, these models easily become non-tractable. In this paper, we provide sufficient conditions for the validity of the first-order approach for twoperiod dynamic moral hazard problems where the agent can save and borrow secretly. To ensure that the agent's problem is jointly concave in effort and asset decisions when facing the optimal contract, we build on the concept of log-convexity. Since this property, unlike convexity, is preserved under multiplication, we are able to separate the assumptions on the distribution function from the assumptions on the agent's preferences, even though the interaction between these two is important for the agent's incentives. We show that the first-order approach is valid if the following conditions hold: i) the agent has nonincreasing absolute risk aversion utility (NIARA), ii) the output technology has monotone likelihood ratios (MLR), and iii) the distribution function of output is log-convex in effort (LCDF). Moreover, under the above three conditions, the optimal contract is monotone in output. We also investigate a few possibilities of relaxing these requirements.
\end{abstract}

Keywords: Moral Hazard, Hidden Savings, First-Order Approach, Log-Convexity.

JEL: C61, D81, D82, D86, E21.

${ }^{*}$ This paper merges and replaces two previous papers: "Optimal Income Taxation and Hidden Borrowing and Lending: The First-Order Approach in Two Periods," by A. Abraham and N. Pavoni, and "The First Order Approach to Moral Hazard Problems with Hidden Saving," by S. Koehne. We are grateful to Ian Jewitt for many useful suggestions. We thank Bruno Biais, Kaiji Chen, Jacques Cremer, Bruno Jullien, Narayana Kocherlakota, Chris Phelan, Bernard Salanié, Ernst-Ludwig von Thadden, and participants at numerous seminars for their comments. Nicola Pavoni thanks the Marie Curie Fellowship MCFI-2000-00689 and the Spanish Ministry of Science and Technology Grant BEC2001-1653.

${ }^{\dagger}$ European University Institute, Florence. E-mail address: Arpad. Abraham@EUI.eu

${ }^{\ddagger}$ University of Mannheim. E-mail address: skoehne@mail.uni-mannheim.de

$\S$ University College London, IFS, and CEPR. E-mail address: n.pavoni@ucl.ac.uk 


\section{Introduction}

The present paper derives general conditions for the validity of the first-order approach (FOA) for twoperiod dynamic moral hazard problems where the agent can save and borrow secretly, and characterizes the optimal contract. We show that the first-order approach is valid if the agent has nonincreasing absolute risk aversion (NIARA) utility, the output technology has monotone likelihood ratios (MLR), and the distribution function of output is log-convex in effort (LCDF). ${ }^{1}$

Recently, dynamic principal-agent models became very popular instruments to study several diverse issues such as design of optimal social insurance schemes (e.g. unemployment insurance, and disability insurance), bank-firm financing relationships, efficient compensation contracts, and optimal capital taxation. Most of these models assume that the agent's consumption-savings decision is observable (and fully contractable) by the principal. However, it is also well-known that this assumption is potentially very dangerous, because if the agent is given a hidden (or not contractable) opportunity to save then he would deviate from the optimal contract by saving (and possibly exerting less effort) (Rogerson, 1985a). Therefore, the possibility of hidden asset accumulation will lead to a different optimal contract. This problem is also relevant empirically, as in most of the above-mentioned applications, the contractability and observability of asset accumulation cannot be guaranteed.

The first-order approach (FOA) replaces the incentive compatibility constraints of the agent by the corresponding first-order necessary conditions from the agent's decision problem. Since the seminal works by Mirrlees (1974) and Holmström (1979) it became obvious that the study of the moral hazard models is much easier if one can rely on the FOA. It becomes even more important to simplify the incentive compatibility constraint in a dynamic environment when the principal faces an additional information problem because the agent has secret access to the credit market. Among others, Rogerson (1985b), Jewitt (1988), and Conlon (2009) provide conditions for the validity of the FOA for the static principal-agent model. Their strategy is to show that when facing the optimal contract, the agent's problem is concave, hence the first-order conditions are actually not only necessary but also sufficient for the optimality of the agent's decisions. This paper follows a similar strategy for dynamic principal agent models with hidden savings.

This extension is important, because it is not known under what conditions the FOA can be applied to principal-agent problems with hidden asset accumulation. In fact, Kocherlakota (2004) finds cases (linearity of both the effort cost and the effort's impact on output) where - although the standard conditions that guarantee the validity of the first-order approach in the static model are verified - the agent's problem is not concave when he is allowed to enter the credit market. Intuitively,

\footnotetext{
${ }^{1}$ A function is called log-convex if the logarithm of that function is convex. Any log-convex functions is convex, but not vice versa.
} 
the non-concavity is a consequence of potential benefits from jointly decreasing effort and increasing savings. The necessary first-order conditions may not capture these second-order gains. In this paper, we provide sufficient conditions for the two-period model under which these second-order gains are small enough and therefore the incentive compatibility constraint for the agent's decisions can be replaced by its necessary and sufficient first-order conditions. In particular, we show that, within the family of non-increasing absolute risk aversion (NIARA) utility functions in consumption, a logconvex distribution function ( $\mathrm{LCDF}$ ) of output guarantees that the FOA is applicable. This is the case whenever the optimal consumption scheme is monotone in output, which is in turn guaranteed by the usual monotone likelihood ratio (MLR) condition together with NIARA utility. We also show that the log-convexity condition we impose on the probability distribution is essentially identical to a requirement on the utility function of leisure. In particular, in standard models of labor supply the requirement would be equivalent to the Frisch elasticity of leisure being less than unity. Our conditions imply that most of the (additively separable) utility functions and many effort specifications used in applications allow for a first-order condition representation of the problem. Further, empirical studies seem to confirm both the NIARA and the Frisch elasticity condition.

Intuitively, the link from the NIARA and LCDF conditions to the concavity of the agent's problem is as follows. First of all, one can show that NIARA utility is equivalent to log-convex marginal utility of consumption. It is also immediate that the two variables subject to private information and under the agent's control - effort and savings - enter multiplicatively into the objective of the agent, hence the aforementioned difficulties in verifying incentive-compatibility. One can show that - after integrating by parts the expected utility of the agent - the key function for the concavity of the agent's problem is the product of the marginal utility of consumption and the cumulative distribution of output. Moreover, this product is multiplied by a negative constant. At this point, the concept of log-convexity proves helpful. In contrast to convexity, log-convexity is not only preserved under summation, but also under multiplication. Therefore, log-convexity of the agent's marginal utility of consumption in combination with log-convexity of the distribution function of output implies that the agent's problem is jointly log-convex (and hence convex) in effort and savings when multiplied by a negative constant. Clearly, this implies that the agent's problem is jointly concave in his decision variables.

We also derive alternative sufficient conditions for the validity of the first-order approach. An important insight of the previous argument is the trade-off between convexity assumptions on the marginal utility of consumption on the one hand and convexity assumptions on the distribution function on the other hand. If one of the two functions is more convex than log-linear, then the assumption on the other function can be weakened. For a large class of utility functions, including CRRA utility, for example, this allows a relaxation of the LCDF condition. Finally, we show how to relax the 
LCDF condition by exploiting the curvature of the consumption scheme. This allows us to validate the first-order approach for some important examples in which the LCDF property and even Rogerson's (1985b) CDF condition fail. From a more general point of view, however, the curvature of the contract is not as helpful as in the standard moral hazard problem, since consumption tends to become less concave in output under hidden savings. ${ }^{2}$

In the present paper we also provide two main characterization results. First, we show that, as opposed to hidden information moral hazard models (see Allen, 1985 and Cole and Kocherlakota, 2001), self-insurance is not optimal in this environment. This result is general in the sense that it does not even require the validity of the FOA. Second, thanks to the use of the first-order approach, we are able to show that, similarly to the pure moral hazard case with observable assets, under the standard monotone likelihood ratio condition, consumption is monotone increasing in income whenever preferences are NIARA.

In addition to allowing a characterization of the optimal contract, finding conditions for the validity of the first-order approach is important for a number of other reasons. First, as explained in Ábrahám and Pavoni (2008), Werning (2001, and 2002), and Kocherlakota (2004), the first-order approach is crucial for being able to write the problem in a tractable recursive form. Second, it can be shown that whenever the first-order approach is valid the optimal tax on asset holdings takes a simple form. In particular, imposing linear taxes on savings which are uniform across ex-post shocks is optimal. ${ }^{3}$ Finally, our conditions for concavity and hence the applicability of the first-order condition approach already proved to be useful in answering several theoretical and applied questions (see Raith, 2008; Ábrahám, Koehne, and Pavoni, 2009; Bertola and Koeniger, 2009; and Chade, 2009).

Unfortunately, our analytical results for the two-period model cannot be easily extended to a framework with more than two periods. ${ }^{4}$ This paper however constitutes a first step toward the analytical study of this class of dynamic moral hazard models. Pointing out the multiplicative structure of the agent's problem and explaining the role played by log-convexity already constitutes an important insight about the problem. In fact, virtually all existing papers that study the moral hazard problem with hidden asset trade or storage analytically, either use very special closed form solutions or use two period models. ${ }^{5}$

\footnotetext{
${ }^{2}$ See Ábrahám, Koehne, and Pavoni (2009) for a more detailed discussion of this insight.

${ }^{3}$ This observation is somewhat implicit in Kocherlakota (2005), and it has been shown to be true for a wide set of assets by Gottardi and Pavoni (2007). In the latter, it is also shown that this simple tax system implies a positive (expected) tax on capital.

${ }^{4}$ For a numerical verication technique of the FOA in more general problems, see Ábrahám and Pavoni (2008).

${ }^{5}$ In a two period principal agent relationship, Bizer and DeMarzo (1999) show that hidden access to the credit market reduces total welfare with respect to the no asset market case. They focus on the possibility of increasing welfare by allowing the entrepreneur to default on the debt. Bisin and Rampini (2006) study the effect of bankruptcy provision, in a
} 
To the best of our knowledge, there is only one other paper which studies systematically the issue of the validity of the first-order approach in this class of models. Williams (2006) gives sufficient conditions for the validity of the FOA for a large set of continuous time principal-agents models. There are a number of important differences between our approach and that of Williams that make the two papers complementary to each other. First, although his conditions are stated for a very large set of models and for any time horizon, they are not satisfied in a context where there is a linear return/storage technology for assets such as assumed here. Second, - as most of the literature with continuous time models - Williams considers a stochastic production technology with normally distributed shocks (the Brownian model). We focus on the two period problem with shocks on a bounded support but allow for virtually any distribution function and any pattern for the likelihood ratios over the support. ${ }^{6}$ This dimension of generality might be of importance, especially when one aims at characterizing the pattern of consumption across income levels (e.g., see Ábrahám, Koehne, and Pavoni, 2009).

The next section presents the model and derives a minimal condition for optimality. Then we introduce the first-order condition approach and provide conditions for the concavity of the agent's problem in the optimum in Section 3. There we also show that - under the same conditions - the optimal consumption scheme is monotonically increasing in output. Section 4 discusses extensions, while Section 5 concludes.

two period model similar to that of Bizer and DeMarzo, where agents have hidden access to insurance contracts and can default on the principal insurer as well. In addition to no-default, we do not allow agents to secretly trade assets other than a risk free bond. Chiappori et al. (1994) and more recently Park (2004) analyze the optimal contract with discrete effort. They find that - under some conditions - a renegotiation-proof contract always implements the minimum level of effort. We consider a continuous-effort model, where the planner can commit not to renegotiate the contract ex post. In fact, Chade (2009) uses the first-order approach we propose here to show that if the agent effort belongs to a continuoum, the planner is typically able to implement a positive level of action even when proposing a renegotiation-proof contract.

Kocherlakota (2004) and Mitchell and Zhang (2009) characterize the optimal UI transfer scheme in an infinite horizon two-output moral hazard model with hidden savings, where agents' preferences are linear in effort, and effort affects linearly job-finding probabilities. Werning (2002) solves analytically a similar two-output model with multiplicative separable CARA utility. In this paper, we consider a two period model which allows for both a general class of preferences and a much more general production technology.

${ }^{6}$ Schattler and Jaryoung (1997) discuss how removing the assumption of Brownian motion in the standard principalagent model in continuos time changes the properties of the optimal contract. Schattler and Jaryoung (1997) also confirm the finding in Mirrlees (1975) and show that for any 'discretization' of the continuous time model, the problem with normally distributed shocks is not well defined. In particular, the optimal contract approximates arbitrarily well the first best allocation by imposing extreme punishments and rewards upon events with very small probability. 


\section{Model}

Consider a relationship between a risk neutral principal/planner and a risk averse agent that lasts for two periods: $t=0,1$. The model builds on the typical dynamic moral hazard problem and assumes that consumption occurs at the beginning of each period (together with the effort decision). ${ }^{7}$

Preferences The agent derives utility from consumption $c_{t} \geq \underline{c} \geq-\infty$ and effort $\infty \geq \bar{e} \geq$ $e_{t} \geq 0$ according to: $u\left(c_{t}\right)-v\left(e_{t}\right)$, where both $u$ and $v$ are strictly increasing and twice continuously differentiable functions, and $u$ is strictly concave whereas $v$ is convex. We normalize $v(0)=0$. The agent's discount factor will be denoted by $\beta>0$.

Production and endowments At date $t=0$, the agent has a fixed endowment $y_{0}$. At date $t=1$, there are $N$ possible output levels $Y:=\left\{y_{1}, \ldots, y_{N}\right\}$ with $y_{i}<y_{i+1}$. The realization $y_{i} \in Y$ is publicly observable, while the probability distribution over $Y$ is affected by the agent's unobservable effort level $e_{0}$ that is exerted at $t=0$. The conditional probabilities are defined by the smooth functions: ${ }^{8} p_{i}\left(e_{0}\right):=\operatorname{Pr}\left\{y=y_{i} \mid e_{0}\right\}$. As in most of the the optimal contracting literature, we assume full support, that is $p_{i}\left(e_{0}\right)>0$ for all $i=1, \ldots, N$, and all $e_{0}$. There is no production or any other action at $t \geq 2$.

Markets At each date, the agent can buy or (short)-sell a risk-free bond $b_{t}$ which costs $q \geq 0$ consumption units today and pays one unit of consumption tomorrow. The agent has no access to any other insurance market other than that delivered by the planner (exclusivity). We assume that asset decisions and consumption levels are private information to the agent.

Given the structure of the problem, the agent will never be able to borrow at $t=1$, hence we have $b_{1} \geq 0$. Monotonicity of preferences guarantees that the agent will not want to leave any positive amount of assets at date 1 either. So, $b_{1}=0$ for all states $i$. Similarly, since $v$ is strictly increasing, $e_{1}=0$ for all states $i$.

Contracts A contract $\mathcal{W}:=(\tau, \sigma)$ is constituted by a transfer scheme $\tau:=\left(\tau_{0},\left\{\tau_{i}\right\}_{i=1}^{N}\right)$, where $\tau_{0}$ and $\tau_{i}$ represent the transfers the individual receives in period $t=0$ and in period $t=1$ conditional on realization $y_{i}$, respectively. To simplify the analysis, we separate the planner's transfer plan from

\footnotetext{
${ }^{7}$ This timing is very common, for example, in the optimal unemployment insurance literature (e.g., Hopenhayn and Nicolini, 1997).

${ }^{8}$ In particular, we require the function $\mathbf{p}: E \rightarrow \Delta^{N}$ to be continuous and continuously differentiable. Here, $\Delta^{N}:=\left\{x \in \mathbb{R}^{N} \mid x \geq 0\right.$ and $\left.\sum_{i} x_{i}=1\right\}$.
} 
the components of the allocation under the agent's control, which are $\sigma:=\left(e_{0}, b_{0}\right)$. Given $\mathcal{W}$, the agent's utility is

$$
U\left(e_{0}, b_{0} ; \tau\right):=u\left(y_{0}+\tau_{0}-q b_{0}\right)-v\left(e_{0}\right)+\beta \sum_{i=1}^{N} p_{i}\left(e_{0}\right) u\left(y_{i}+\tau_{i}+b_{0}\right) .
$$

Recall that a key assumption in our model is that the planner cannot observe how the agent allocates his income $y_{0}+\tau_{0}$ between consumption $c_{0}$ and asset accumulation $q b_{0}$. As usual, to guarantee solvency of the agent for every contingency, we impose the 'natural' borrowing limit: $b_{0} \geq \underline{c}-\min _{i=1, . . N}\left\{y_{i}+\tau_{i}\right\} .{ }^{9}$

The risk neutral planner faces the same credit market as the agent, therefore her discount rate is $q$. Her preferences/profits are

$$
V\left(e_{0}, b_{0} ; \tau\right):=-\tau_{0}+q \sum_{i=1}^{N} p_{i}\left(e_{0}\right)\left(-\tau_{i}\right)
$$

Efficiency An optimal contract is a contract that solves ${ }^{10}$

$$
V\left(U_{0}\right):=\max _{\mathcal{W}} V\left(e_{0}, b_{0} ; \tau\right),
$$

subject to the participation constraint

$$
U\left(e_{0}, b_{0} ; \tau\right) \geq U_{0}
$$

and the incentive compatibility constraint

$$
\left(e_{0}, b_{0}\right) \in \arg \left\{\max _{e, b} U(e, b ; \tau) \text { s.t. } e \geq 0, y_{0}+\tau_{0}-\underline{c} \geq q b \geq-q \min _{i}\left\{y_{i}+\tau_{i}-\underline{c}\right\}\right\} .
$$

We will denote this problem as $(\mathbf{P})$. In order to make the problem of some interest, we assume that $U_{0}>-\infty$.

Note that there is indeterminacy in the contract between $\tau_{0}$ and $b_{0}$. The planner can implement the same allocation to the agent with a contract $\left\{\tau_{0},\left\{\tau_{i}\right\}_{i=1}^{N}, e_{0}, b_{0}\right\}$ and with a contract $\left\{\tau_{0}-\varepsilon,\left\{\tau_{i}+\varepsilon / q\right\}_{i=1}^{N}, e_{0}, b_{0}-\varepsilon / q\right\}$. In other words, since the planner and the agent face the same return in the credit market, there is a continuum of optimal contracts. In this paper, without a loss of generality, we will study the one specific optimal contract which implements $b_{0}=0$. Because of these

\footnotetext{
${ }^{9}$ The enforceability of the repayment of debt obtained through anonymous credit lines is an important and delicate issue, which is common to many environments and that we do not address here. With minor modifications to the analysis, one could just impose $b_{0} \geq 0$, which could be enforced in an anonymous credit market. In this this case, asset accumulation could also be interpreted as a private storage technology.

${ }^{10}$ Existence can be shown, for example, by a simple extension to Grossman and Hart (1983).
} 
observations, we will sometimes refer to the combination of $e_{0}$ and $c_{i}:=y_{i}+\tau_{i}, i=0,1, \ldots, N$, as a contract.

We can interpret this setup in several different ways. For example, the previously described principal-agent relationship can be interpreted as an optimal tax/transfer provision problem (social insurance). The principal is a benevolent social planner whose objective is to maximize the welfare of the citizens. The (small open) economy contains a continuum of ex ante identical agents who face the above contract and can influence their date 1 income realizations by working hard or shirking. The planner offers a tax/transfer system to insure them against idiosyncratic risk and, at the same time, provide them appropriate incentives for working hard. In this case, setting $U_{0}$ such a way that $V\left(U_{0}\right)=0$ would be the socially optimal allocation. The objective (2) can be interpreted as the social planner's dynamic budget constraint; by the law of large numbers $V\left(U_{0}\right)=0$ is equivalent to an (intertemporally) balanced budget requirement. In what follows, we will take the value of ex-ante utility $U_{0}$ as given and derive the optimal tax/transfer scheme by minimizing expected discounted costs.

This framework may also describe a private insurance relationship, where $y_{0}$ is the agent's (verifiable) initial net wealth and he can affect future outcomes by his action $e_{0}$. The insurance company is the principal, who offers a contract consisting of an initial fee $\left(\tau_{0}\right)$ and a (net) insurance payment $\left(\tau_{i}\right)$ dependent on the realized state. In this case, if we set $U_{0}$ such that $V\left(U_{0}\right)=0$, the insurance contract will deliver zero profits to the insurance company. When designing the conditions of the contract, the insurer has to take into account that the agent can secretly influence the likelihood of the different events. In addition, in our environment, the insurer cannot observe the agent's consumption either, therefore the optimal insurance contract has to take into account that the agent is able to transfer resources intertemporally through the credit market.

Another interpretation is a two-period compensation contract, where $y_{i}$ is the surplus which can be divided by a risk-neutral owner/shareholder and a risk-averse worker/manager. In this case, $y_{i}+\tau_{i}$ is the wage of the worker in a given state and date, while $V\left(U_{0}\right)$ is the profit of the owner. Here, again, the wages have to provide the right incentives for the agent to exert high effort. Moreover, high punishments (low consumption levels) for low second period surpluses may not be incentive compatible, because the worker can save against them at date $t=0$.

The main implications of this framework are known when hidden borrowing and lending are not allowed. We will briefly review them below. However, Rogerson (1985a) has shown for that setup that the agent is left with incentives to save in the optimal contract. This implies that the optimal allocation of the present model is different from the one where asset accumulation is observable and contractable or not allowed. Moreover, in all of the three above examples, private (non-observable, 
non-contractable or non-taxable) savings are empirically relevant. Neither insurance companies nor shareholders/owners can control the agent's consumption saving decision, however their wealth level affects the effectiveness of the incentive scheme. Also, typically governments cannot have full control over agents' consumption saving decisions either, because agents can keep their savings in low-interest (and not observable) instruments such as local and foreign currency or they can have access to foreign accounts.

Finally, as we discuss in the Conclusions section, it is not difficult to show that our analysis would carry over to the case where the agent has a private storage technology that allows $q$ units of consumption at time 0 to be transformed into one unit of consumption at time 1 . This is the case, because in this model the agent's relevant deviation is to transfer resources from period 0 to period 1 , hence borrowing constraints matter less.

\subsection{Preliminary Characterization and Self-insurance}

Under very general conditions (in particular without requiring that the first-order approach is valid) one can prove that transfers $\tau_{i}$ cannot uniformly increase with $y_{i}{ }^{11}$

Proposition 1 (Minimal Insurance) If $u$ is unbounded below or above, and $\underline{c}>-\infty$, then $\tau_{i}$ cannot be monotone increasing (not even weakly increasing) with $y_{i}$ for all $i$.

The intuition is as follows. A transfer scheme with $\tau_{i}$ increasing for all $i$ cannot be optimal, since the planner could twist the scheme so that to decrease $\tau_{i}$ in states with large $y_{i}$ and increase it in bad states (states with low $y_{i}$ ). Since the agent is risk averse and the planner is risk neutral, we expect the planner to gain by absorbing some of the income risk the agent faces. In particular, the planner should be able to provide more insurance to the agent than he can achieve without the planner's participation.

Proposition 1 has a somewhat important consequence. Allen (1985) and Cole and Kocherlakota (2001) study the effect of hidden asset accumulation in a hidden information model. They find that the constrained efficient allocation does not differ from that in a pure bond economy, i.e., the allocation the agents could obtain by insuring themselves through borrowing and lending, without the planner's provision of additional insurance. In terms of our optimal taxation interpretation, this result would imply that the planner has no role in enhancing welfare, as the optimal allocation will provide no additional insurance to the agents compared to a pure bond economy.

Note that a pure bond economy would correspond to a situation of constant $\tau_{i}=\tau$ for $i=1,2, . . N$. Proposition 1 shows that this is cannot be the case for the moral hazard model.

\footnotetext{
${ }^{11}$ All the proofs not shown in the main text can be found in the Appendix.
} 
Corollary to Proposition 1 (Impossibility of Self-Insurance) Under the assumptions in Proposition 1, the optimal contract never delivers the self insurance allocation.

Since the contract $\tau_{i} \equiv \tau$ is available to the planner, an equivalent way of stating the result is as follows. If we let $\underline{U}_{0}$ be the utility value obtainable by the agents under self-insurance, we have $V\left(\underline{U}_{0}\right)>0$. What is then the key difference between the hidden information models and the moral hazard models that generates these contrasting implications? As it is clear from our line of proof, the full support assumption plays a major role in the result. Under this condition, the agent has incomplete control over the realizations $y_{i}$. Hence, the planner can implement schemes that impose a tax payment in some states and a transfer in others in such a way that the agent is not able to avoid paying the taxes with certainty. Clearly, this result extends to any multi-period setting. ${ }^{12}$

It is very unlikely that we would be able to characterize the optimal contract much further analytically without the use of the first-order approach. In the next section, we will introduce this approach and provide sufficient conditions for its validity.

\section{The First-Order Approach}

It is not difficult to see that the incentive constraint (5) describes a complicated set of constraints: it is equivalent to a bidimensional continuum of inequalities. The first-order approach replaces (5) by the first-order conditions of the agent's maximization problem with respect to $e_{0}$ and $b_{0}$. This strategy brings the number of inequality constraints down to only two.

In what follows, we will assume interiority of the optimal contract, that is we assume that the original problem $(\mathbf{P})$ has a solution such that all consumption levels are strictly above $\underline{c}$ in both dates and for all states and that $e_{0}>0$. It is easy to see that the moral hazard problem is not interesting if the optimal effort is the minimal effort. ${ }^{13}$ We will follow Rogerson (1985b) and replace the incentive

\footnotetext{
${ }^{12}$ Moreover, consistently with the previous intuition, in Ábrahám and Pavoni (2006) we show that, in fact, the fullsupport assumption is not required. It suffices to exclude the possibility of distributions over $Y$ which are degenerate at one state $i$ for some $e$.

${ }^{13}$ Interiority may be required for a more technical reason. As emphasized by Mirrlees (1975), when the solution to the original problem is at the corner then the first-order approach might fail to deliver even necessary conditions for an optimum. In our case, we could guarantee interiority in effort by assuming that $e$ is taken within an open set. Alternatively, we could avoid the complications due to the corner solution by assuming that $v^{\prime}(0)=0$. Interiority with respect to consumption can be guaranteed by imposing that consumption is chosen within an open interval (as in Grossman and Hart, 1983), or by requiring that $\lim _{c \rightarrow \underline{c}} u^{\prime}(c)=+\infty$.
} 
constraint with the following necessary conditions for optimality of the decisions for $e_{0}$ and $b_{0}(=0)$ :

$$
\begin{aligned}
& U_{e}\left(e_{0}, 0 ; \tau\right) \geq 0 \\
& \text { and } \\
& U_{b}\left(e_{0}, 0 ; \tau\right) \leq 0 .
\end{aligned}
$$

The intuition for the above conditions is simple. There are two key restrictions we impose on the contract. First, we guarantee that the agent is not willing to reduce his effort level or shirk. The second condition is the usual inequality version of the Euler equation: we require the agent not willing to save. Clearly, both conditions have a local nature.

Notice, that both above constraints depend on the agent's equilibrium choices of consumption and effort alone. Given our normalization on $b_{0}=0$, it will indeed be convenient to describe the principalagent relationship as one where the planner decides directly the consumption level of the agent at each state. We define $\mathbf{c}:=\left\{c_{i}\right\}_{i=0}^{N}$, where $c_{i}:=y_{i}+\tau_{i}$, and rewrite the planner's problem as

$$
\max _{e_{0}, \mathbf{c}} y_{0}-c_{0}+q \sum_{i=1}^{N} p_{i}\left(e_{0}\right)\left(y_{i}-c_{i}\right),
$$

subject to $c_{i} \geq \underline{c}, e_{0} \geq 0$, the relaxed incentive constraint (6), which can be written as follows:

$$
\begin{aligned}
& -v^{\prime}\left(e_{0}\right)+\beta \sum_{i} p_{i}^{\prime}\left(e_{0}\right) u\left(c_{i}\right) \geq 0, \\
& q u^{\prime}\left(c_{0}\right)-\beta \sum_{i} p_{i}\left(e_{0}\right) u^{\prime}\left(c_{i}\right) \geq 0,
\end{aligned}
$$

and the participation constraint (4)

$$
u\left(c_{0}\right)-v\left(e_{0}\right)+\beta \sum_{i=1}^{N} p_{i}\left(e_{0}\right) u\left(c_{i}\right) \geq U_{0} .
$$

This problem will be denoted by $(\mathbf{R})$. We refer to this second problem as relaxed, because the set of contracts that satisfy the constraints of $(\mathbf{R})$ contains the set of contracts that satisfy the constraints of the original problem $(\mathbf{P})$. The remainder of this section is devoted to provide conditions under which the solution to the relaxed problem $(\mathbf{R})$ is identical to the solution of the original (unrelaxed) problem (P) given by conditions (3) to (5).

Until now, there were not known any conditions under which the above first-order conditions are necessary and sufficient for incentive compatibility. In Ábrahám and Pavoni (2008), we approach this issue by a numerical ex-post verification procedure for generic multi-period problems. However, that procedure requires a numerical solution of the problem. 
Williams (2006) provides sufficient conditions for concavity for a class of continuous time models with and without hidden savings. The key distinction between his approach and the one followed here, is that he provides sufficient conditions for the cases where either the price of the bond $q$ is not constant but a convex function of $b$, or $q$ is constant but the agent's utility directly depends on wealth in a strictly concave way.

We will find sufficient conditions for concavity in two steps. First, we will characterize the 'relaxed' optimal contract $(\mathbf{R})$ by imposing the agent's first-order conditions instead of (5). Then, we prove that, under appropriate conditions, in the relaxed optimal contract date- 1 consumption $c_{i}$ is changing monotonically with the agent's income/output $y_{i}$. Finally, we show that, under somewhat stricter conditions, monotonicity of consumption implies that the agent's problem is concave at the relaxed optimal contract. This will imply that, under this set of stricter conditions, our use of the first-order condition approach is actually justified, as the solution to $(\mathbf{R})$ also constitutes a solution to $(\mathbf{P})$.

\subsection{Monotonicity of Consumption}

We start by analyzing the properties of consumption based on the relaxed problem $(\mathbf{R})$. We first introduce some well known properties of the probability shifting functions $\mathbf{p}$ and the utility function, which we will use extensively later. We will make use of the following assumptions.

NIARA. The utility function $u$ exhibits non-increasing absolute risk aversion, that is, the ratio $a(c):=-\frac{u^{\prime \prime}(c)}{u^{\prime}(c)}$ is non-increasing in $c$.

MLR. The probability shifting function $\mathbf{p}$ has the monotone likelihood ratio property, that is, for each $e \geq 0$ the ratio $\frac{p_{i}^{\prime}(e)}{p_{i}(e)}$ is non-decreasing in $i$.

We can now state our second characterization result.

Proposition 2 (Monotonicity) Assume NIARA and let $\left(\mathbf{c}, e_{0}\right)$ be a solution to (R). (i) Either $c_{i}=\underline{c}$, or $c_{i}$ moves together with the likelihood ratio $\frac{p_{i}^{\prime}\left(e_{0}\right)}{p_{i}\left(e_{0}\right)}$. (ii) Under MLR, $c_{i}$ is nondecreasing in $i$ for $i=1,2, . ., N$.

Proof. Consider problem $(\mathbf{R})$, and denote by $\mu, \xi$ and $\lambda$ the Kuhn-Tucker multipliers associated to the constraints (7), (8), and (4) respectively. By standard conditions, they are all nonnegative. The necessary conditions for optimality (with respect to $c_{0}$ and $c_{i}$ ) are:

$$
\frac{1}{u^{\prime}\left(c_{0}\right)} \geq \lambda+\xi \frac{q u^{\prime \prime}\left(c_{0}\right)}{u^{\prime}\left(c_{0}\right)}, \text { with equality if } c_{0}>\underline{c} .
$$

And, for $i=1, . ., N$, we either have $c_{i}=\underline{c}$, or:

$$
\frac{q}{\beta u^{\prime}\left(c_{i}\right)}=\lambda+\mu \frac{p_{i}^{\prime}\left(e_{0}\right)}{p_{i}\left(e_{0}\right)}-\xi \frac{u^{\prime \prime}\left(c_{i}\right)}{u^{\prime}\left(c_{i}\right)}=\lambda+\mu \frac{p_{i}^{\prime}\left(e_{0}\right)}{p_{i}\left(e_{0}\right)}+\xi a\left(c_{i}\right) .
$$


Note that from (10) and $\mu \geq 0$, the expression $\frac{q}{\beta u^{\prime}\left(c_{i}\right)}-a\left(c_{i}\right) \xi$ must move together with the likelihood ratio $\frac{p_{i}^{\prime}\left(e_{0}\right)}{p_{i}\left(e_{0}\right)}$. By concavity of $u$ and NIARA, both $\frac{1}{u^{\prime}}$ and $-a\left(c_{i}\right)=\frac{u^{\prime \prime}}{u^{\prime}}$ increase with $c_{i}$. Since $\xi \geq 0$, consumption must move with $i$ in the same direction as $\frac{p_{i}^{\prime}\left(e_{0}\right)}{p_{i}\left(e_{0}\right)}$. Q.E.D.

Proposition 2 replicates a standard result in the contract theory literature with no (or observable and fully contractable) access to the credit market. ${ }^{14}$ In terms of the compensation contracts example, this result says that the wages are weakly increasing with the observed output of the agent. More generally, the first part of Proposition 2 emphasizes that in our environment consumption varies across states in proportion to $\frac{p_{i}^{\prime}\left(e_{0}\right)}{p_{i}\left(e_{0}\right)}$ alone. That is, consumption only responds to the informational content of the outcome realization $y_{i}$ on the effort level $e_{0}$. Note that this contrasts the self-insurance allocation where, since taxes $\tau$ are constant across states, $c_{i}$ moves one to one with $y_{i}$ regardless of the informational content of income levels. All the above properties are strict as long as MLR holds in a strict version and both $\mu$ and $\xi$ are strictly positive. The next Lemma establishes this latter fact when $e_{0}>0$.

Lemma 1 Assume NIARA and let $\left(\mathbf{c}, e_{0}\right)$ be a solution to $(\mathbf{R})$. Then (i) If $c_{i}>\underline{c}$ for $i=1,2, \ldots N$ then $U_{e}=U_{b}=0$, that is, constraints (7) and (8) are both satisfied with equality. (ii) If both $c_{i}>\underline{c}$ for all $i$ and $e_{0}>0$, then both $\mu>0$ and $\xi>0$. (iii) If $u$ satisfies the Inada condition $\lim _{c \rightarrow \underline{c}} u^{\prime}(c)=\infty$, then (i) holds under NIARA without the interiority requirement on consumption.

Of course, if $u$ is unbounded below or $\underline{c}=-\infty$, the interiority condition $c_{i}>\underline{c}$ can be guaranteed a priori. Lemma 1 shows that, whenever we are using the first-order approach, under mild regularity conditions we can in fact impose (6) with equality. If, in addition, we are looking for interior solutions for effort, then the multipliers associated with the first-order incentive constraints are positive. The following remark shows that the multiplier associated with the participation constraint is positive whenever we have an interior solution for $c_{0}$.

Remark 1 It is easy to see that whenever $c_{0}>\underline{c}$ we have $\lambda>0$. This is so since whenever $U\left(e_{0}, 0 ; \tau\right)>$ $U_{0}$ the planner could always reduce $c_{0}$. This modification of the contract does not affect the effort incentive constraint, relaxes the Euler equation, and increases the planner's net returns.

We now move to the concavity of the agent's problem.

\footnotetext{
${ }^{14}$ Note that the properties of the optimal contract, in the case when the agent has no access to the credit market, can be recovered from the above optimality conditions by assuming $\xi=0$.
} 


\subsection{Sufficient Conditions for Global Concavity of the Agent's Problem}

Lemma 1 guarantees that the first-order condition (6) in the relaxed problem (R) holds with equality. Whenever the solution to the original problem $(\mathbf{P})$ is interior, a necessary condition for incentive compatibility is to have (6) satisfied with equality, of course. Since (R) is a relaxed problem, the value associated to it cannot be lower than that associated to $(\mathbf{P})$. Therefore, if we show that the contract solving $(\mathbf{R})$ is actually incentive compatible, then we have in fact derived the optimal contract. We hence say that the first-order approach is valid whenever a solution to the relaxed problem $(\mathbf{R})$ delivers a correct solution to the original problem $(\mathbf{P})$. If the agent's problem is globally jointly concave in $e$ and $b$ when facing the optimal transfer scheme $\tau$, the use of the first-order approach is valid as the first-order conditions of the agent's problem become sufficient conditions for incentive compatibility. Recall that, given $\tau$, the utility of the agent for each choice $(e, b)$ is

$$
U(e, b ; \tau)=u\left(y_{0}+\tau_{0}-q b\right)-v(e)+\beta \sum_{i=1}^{N} p_{i}(e) u\left(y_{i}+\tau_{i}+b\right),
$$

Global concavity is obviously guaranteed if the associated Hessian matrix is negative semidefinite for all $e$ and $b$, where the Hessian is given by $\left(c_{i}\right.$ is defined by $\left.y_{i}+\tau_{i}\right)$

$$
H:=\left[\begin{array}{cc}
-v^{\prime \prime}(e)+\beta \sum_{i} p_{i}^{\prime \prime}(e) u\left(c_{i}+b\right) & \beta \sum_{i} p_{i}^{\prime}(e) u^{\prime}\left(c_{i}+b\right) \\
\beta \sum_{i} p_{i}^{\prime}(e) u^{\prime}\left(c_{i}+b\right) & q^{2} u^{\prime \prime}\left(c_{0}-q b\right)+\beta \sum_{i} p_{i}(e) u^{\prime \prime}\left(c_{i}+b\right)
\end{array}\right] .
$$

By the concavity of the utility function $u$, it is easy to see that problem is concave in $b$ alone. Rogerson (1985b) shows that if the distribution function is convex in effort (CDF), ${ }^{15}$ the problem is also concave in $e$ alone, whenever the consumption scheme is monotone in output (which is guaranteed by MLR and NIARA) ${ }^{16}$ Unfortunately, the CDF and MLR conditions will not guarantee the concavity of the agent's problem jointly in $(e, b)$; that will require a stronger assumption.

Joint concavity requires that the Hessian is negative semidefinite. This is not true in general, not even under CDF and MLR. ${ }^{17}$ The previous discussion however guarantees that, under NIARA, CDF and MLR, both entries on the main diagonal of $H$ are nonpositive. $H$ is hence negative semidefinite if and only if $\operatorname{det} H \geq 0$. Now, observe the off-diagonal elements: $\beta \sum_{i} p_{i}^{\prime}(e) u^{\prime}\left(c_{i}+b\right)$. They measure

\footnotetext{
${ }^{15}$ The definition is standard. The functions $\left\{p_{i}(e)\right\}_{i=1}^{N}$ satisfy the CDF condition if $F_{i}^{\prime \prime}(e)$ is non-negative for every $e$ and $i \leq N$, where $F_{i}(e)=\sum_{k=1}^{i} p_{k}(e)$.

${ }^{16}$ Note, importantly, that monotonicity of consumption is not affected by borrowing or lending, since the bond pays equal amounts in each state $i$.

${ }^{17}$ Kocherlakota (2004), provides a 'linear-linear' counter-example where he assumes that $p_{i}^{\prime \prime}(e)=v^{\prime \prime}(e)=0$, and therefore

$$
\operatorname{det} H=-\left(\beta \sum_{i} p_{i}^{\prime}(e) u^{\prime}\left(c_{i}+b\right)\right)^{2}<0
$$
}


the gains from joint deviations of postponing consumption and reducing effort (saving and shirking). Intuitively, the agent's problem is concave if the gains from these joint deviations are sufficiently small compared to the losses induced by moving away from the optimal levels of $e_{0}$ and $b_{0}$ (captured by the main diagonal).

Note that the off-diagonal elements of the Hessian are determined only by the date- 1 consequences of deviations. Therefore, to validate the first-order approach, we can restrict attention to date 1 as the next result shows. ${ }^{18}$

Remark 2 The agent's decision problem is concave in $(e, b)$ if his date- 1 utility

$$
(e, b) \mapsto \sum_{i=1}^{N} p_{i}(e) u\left(y_{i}+\tau_{i}+b\right)
$$

is concave in $(e, b)$.

By focusing on the date-1 effect, we ignore the curvature generated by the effort disutility function $v$ and by the impact of saving on date- 0 utility. In principle, one could obtain more general results by including these two effects. We will discuss this issue in a bit more detail below (see Section 3.2.1).

To establish concavity of (13), and hence the validity of the first-order approach, we introduce the following property.

LCDF. The distribution function of output, $F_{i}(e)=\sum_{k=1}^{i} p_{k}(e)$, is log-convex in effort $e$ for all $i$.

A function $f: \mathbb{R} \rightarrow \mathbb{R}_{++}$is called log-convex if the logarithm of $f$ is convex. Given differentiability, $f$ is obviously log-convex if and only if $f^{\prime \prime} f / f^{\prime 2} \geq 1$. Any log-convex functions is convex, but not vice versa. Thus, a necessary but not sufficient condition for LCDF is that the distribution function, $F_{i}(e)$, is convex in effort $e$ for all $i$. In other words, LCDF tightens the CDF condition from Mirrlees (1979) and Rogerson (1985b). Since $F_{i-1}(e)$ equals $1-P\left(y>y_{i} \mid e\right)$, LCDF implies that the probability $P\left(y>y_{i} \mid e\right)$ that output is larger than some level $y_{i}$ is strongly concave in effort $e$ for all values of $y_{i}$.

Now we are ready to formulate the following main result: MLR, NIARA and LCDF validate the first-order approach.

Theorem 1 (FOA) Let $(\tau)$ be a transfer scheme that solves $(\mathbf{R})$. Suppose MLR, NIARA and LCDF. Then, given $(\tau)$, the agent's decision problem is concave. Hence, $(\tau)$ solves the unrelaxed problem $(\mathbf{P})$.

\footnotetext{
${ }^{18}$ It should be clear that we can extend our condition to allow effort to enter in a non-separable way into date- 0 utility. It can be shown that Theorem 1 below remains valid as long as $u$ is concave and supermodular in $\left(c_{0}, e_{0}\right)$. Details are available upon request.
} 
Proof. By Remark 2, it is sufficient to establish concavity of the agent's consumption utility at date-1. Using summation by parts, the latter can be rewritten as

$$
\sum_{i=1}^{N} p_{i}(e) u\left(c_{i}+b\right)=F_{N}(e) u\left(c_{N}+b\right)-\sum_{i=1}^{N-1} F_{i}(e)\left[u\left(c_{i+1}+b\right)-u\left(c_{i}+b\right)\right],
$$

where $F_{i}(e)=\sum_{k=1}^{i} p_{k}(e), i=1, \ldots, N$, denotes the distribution function of output. Since $u\left(c_{N}+b\right)$ is concave in $b$ and $F_{N}(e)=1$, it remains to show that the function

$$
\sum_{i=1}^{N-1} F_{i}(e)\left[u\left(c_{i+1}+b\right)-u\left(c_{i}+b\right)\right]
$$

is convex in $(e, b)$. Note that MLR and NIARA imply $c_{i+1} \geq c_{i}$ for all $i$. If for some index $i$, we have $c_{i+1}=c_{i}$ and hence $u\left(c_{i+1}+b\right)=u\left(c_{i}+b\right)$, then we ignore that index. ${ }^{19}$ This will be without loss of generality as it will not change the concavity properties of our mapping. So, assume $c_{i+1}>c_{i}$.

A key step in our proof is to observe that the terms

$$
F_{i}(e)\left[u\left(c_{i+1}+b\right)-u\left(c_{i}+b\right)\right]
$$

are multiplicatively separable in $e$ and $b$; effort $e$ only enters into the distribution function $F_{i}(e)$, whereas saving $b$ only affects the differences $u\left(c_{i+1}+b\right)-u\left(c_{i}+b\right)$.

Another important step is to see that the NIARA property generates a strong convexity condition for the differences $u\left(c_{i+1}+b\right)-u\left(c_{i}+b\right)$.

Lemma 2 The utility function $u$ exhibits the NIARA property if and only if $u\left(c_{i+1}+b\right)-u\left(c_{i}+b\right)$ is log-convex in $b$ for all $c_{i+1}>c_{i}$.

To prove that statement, it is crucial to note that the agent is more prudent than risk-averse under NIARA (Gollier 2001, p. 52). See the appendix for details.

In addition to the log-convexity property of $u\left(c_{i+1}+b\right)-u\left(c_{i}+b\right)$, we have log-convexity of the distribution function $F_{i}(e)$ by assumption. Therefore, using the multiplicative separability of (15), and the fact that log-convexity (unlike convexity) is preserved under multiplication, we conclude that (15) is log-convex in $(e, b)$. Hence, $(15)$ is convex in $(e, b)$. Since convexity is preserved under summation, this implies that $(14)$ is convex in $(e, b)$. And this completes the proof of the theorem. Q.E.D.

To understand the link from the conditions in Theorem 1 to the second-order effects of joint deviations, it is a useful starting point to study the effect of the agent's two decision variables separately. The agent's effort choice, first of all, determines the probability distribution over date-1 consumption.

\footnotetext{
${ }^{19}$ Note that, because of the full-support assumption, we have $F_{i}(e)>0$ for all $i, e$.
} 
Since consumption is monotonic under MLR and NIARA (Proposition 2), we know that higher effort shifts the distribution towards higher levels of consumption. Equivalently, lower effort shifts the distribution towards 'punishments', i.e., lower consumption levels. The marginal changes in these probabilities are captured by the first-derivative of the distribution function with respect to effort. Essentially, the LCDF assumption ensures that the 'marginal probability of being punished' increases quickly as the agent reduces effort. In other words, there is a second-order effect along this dimension that hurts the agent.

As a second step, it is important to understand the role of the agent's savings level. Note that higher saving generates insurance against punishments: the utility difference between any two transfer levels will be reduced if saving is increased. However, under our assumptions, such differences are decreasing at a rate that reduces quickly: Lemma 2 states that, under NIARA, the utility differences are log-convex in savings. This again captures a second-order effect that hurts the agent.

The two previous observations show that second-order effects of one-dimensional deviations are rather painful for the agent. To see that this is sufficient to rule out two-dimensional deviations, it is crucial to observe that the 'punishment functions' $F_{i}(e)\left[u\left(c_{i+1}+b\right)-u\left(c_{i}+b\right)\right]$ are multiplicatively separable in the agent's decision variables: The distribution function, $F_{i}(e)$, depends only on the agent's effort $e$, while the utility differences, $u\left(c_{i+1}+b\right)-u\left(c_{i}+b\right)$, depend only on the savings level $b$. The multiplicative separability implies that the Hessian of the 'punishment functions' takes a particularly simple form. Therefore, by having the right convexity properties of the two factors (log-convexity of both $F_{i}(e)$ and $u\left(c_{i+1}+b\right)-u\left(c_{i}+b\right)$ ), we can be sure that the off-diagonal of the Hessian is sufficiently small compared to the main diagonal, so that joint deviations are not attractive.

While NIARA is a common property, LCDF is less well-known. As argued above, LCDF states that the marginal returns to effort are strongly decreasing in a particular sense. To clarify this property, we provide the following examples.

Example 1 (Two outputs) Consider the case with two possible outputs, $y_{L}<y_{H}$, and associated probabilities $p_{L}(e)=1-p(e), p_{H}(e)=p(e)$, for some increasing function $p$ with $0<p(e)<1$. Since $p$ is increasing, MLR is satisfied. LCDF is equivalent to the log-convexity of $1-p(e)$. This is equivalent to the condition

$$
\frac{-p^{\prime \prime}(e)(1-p(e))}{p^{2}(e)} \geq 1 \text { for all } e .
$$

A linear (or convex) function $p$ would clearly violate this requirement. Therefore, LCDF requires that there is enough concavity in the probability shifting function. One class of examples that satisfy this property is given by $p(e)=1-\exp (-f(e))$, where $f:[0, \infty) \rightarrow(0, \infty)$ is increasing and concave. 
Example 2 Rogerson's (1985b) paper contains the following distribution function that is convex in effort and satisfies MLR:

$$
F_{i}(e)=\left(y_{i} / y_{N}\right)^{e}, \quad e \in[0, \infty)
$$

This distribution function is not only convex in $e$, but in fact even log-convex. Note

$$
\log \left(F_{i}(e)\right)=e \log \left(\frac{y_{i}}{y_{N}}\right)
$$

which shows that $\log \left(F_{i}\right)$ is linear in $e$ for all $i$.

\subsubsection{A 'Labor Supply' Interpretation of LCDF}

In this subsection, we relate the LCDF condition to a preference parameter of the agent. This parameter has the interpretation as the Frisch elasticity of leisure in related models.

It should be clear from our previous analysis that the log-convexity condition on the distribution function can be relaxed by imposing some strong convexity conditions on the disutility of effort $v(e)$. When $v$ is strongly convex, we can make an appropriate change in variables so that the new disutility function becomes linear. If the distribution function is log-convex in effort with respect to the new unit of measurement, then the FOA is valid (given MLR and NIARA).

More formally, the first-order approach is valid for any couple of functions $\hat{F}_{i}$ and $\hat{v}$ (defined over values of $h$ ), such that the change in variable $e:=\hat{v}(h)$ and $F_{i}(e):=\hat{F}_{i}\left(\hat{v}^{-1}(e)\right)$ leads to log-convex functions $F_{i}(e)$ for all $i$. Now, notice that $F_{i}(e)$ is log-convex if and only if for all $e$

$$
\frac{F_{i}^{\prime \prime}(e) F_{i}(e)}{\left(F_{i}^{\prime}(e)\right)^{2}} \geq 1
$$

Using the definition of $F_{i}$ and the identities

$$
\left(\hat{v}^{-1}\right)^{\prime}(e)=\frac{1}{\hat{v}^{\prime}(h)} \quad, \quad\left(\hat{v}^{-1}\right)^{\prime \prime}(e)=-\frac{\hat{v}^{\prime \prime}(h)}{\left(\hat{v}^{\prime}(h)\right)^{3}}
$$

we can express condition (16) in terms of the variable $h$ as follows:

$$
\frac{\hat{F}_{i}^{\prime \prime}(h) \hat{F}_{i}(h)}{\left(\hat{F}_{i}^{\prime}(h)\right)^{2}}-\frac{\hat{F}_{i}(h)}{\hat{F}_{i}^{\prime}(h)} \frac{\hat{v}^{\prime \prime}(h)}{\hat{v}^{\prime}(h)} \geq 1 .
$$

To link this condition to a preference parameter, we would like to shut down all effects that are related to the curvature of the distribution function. Therefore, we impose some further structure on the problem.

Let $\left(\pi_{1 h}, \ldots, \pi_{n h}\right),\left(\pi_{1 l}, \ldots, \pi_{n l}\right)$ be two probability distributions on $\left\{y_{1}, \ldots, y_{N}\right\}$ such that $\pi_{i h} / \pi_{i l}$ is nondecreasing in $i$. (This implies that $\pi_{h}$ first-order stochastically dominates $\pi_{l}$.) Let the output probabilities be defined as

$$
\hat{p}_{i}(h):=h \pi_{i h}+(1-h) \pi_{i l} .
$$


This requirement is called the spanning condition with dominance, and it has been used in the literature in order to guarantee monotonicity of the consumption scheme in the standard moral hazard model with observable asset accumulation (see Grossman and Hart, 1983; and Atkeson, 1991). The intuition is simple: we have two base distributions given by the vectors $\pi_{l}$ and $\pi_{h}$, in such a way that the distribution given by $\pi_{h}$ first-order stochastically dominates $\pi_{l}$. Intuitively, by exerting higher effort the agent shifts the probability distribution of future outcomes towards the 'better' distribution.

The structure imposed by (19) implies that the distribution function $\hat{F}_{i}(h)$ is linear in $h$ for all $i$ :

$$
\hat{F}_{i}(h)=\sum_{k=1}^{i} \hat{p}_{k}(h)=h \sum_{k=1}^{i} \pi_{k h}+(1-h) \sum_{k=1}^{i} \pi_{k l} .
$$

Hence, the first term in condition (18) disappears. For the second term, we have $-\hat{F}_{i}(h) / \hat{F}_{i}^{\prime}(h) \geq 1-h$. Therefore, condition (18) holds and hence the FOA is valid if the function $\hat{v}$ satisfies the following property:

$$
(1-h) \frac{\hat{v}^{\prime \prime}(h)}{\hat{v}^{\prime}(h)} \geq 1 .
$$

Given the linear relationship between $h$ and (expected) earnings, we can interpret $h$ as labor supply and $1-h$ as leisure. Similarly, we can interpret the function $g(1-h):=-\hat{v}(h)$ as the agent's utility of leisure. Notice that (21) is equivalent to the following property:

$$
-(1-h) \frac{g^{\prime \prime}(1-h)}{g^{\prime}(1-h)} \geq 1 \text {. }
$$

In standard models of labor supply, $\eta^{l}:=-\frac{g^{\prime}(1-h)}{g^{\prime \prime}(1-h)(1-h)}$ is shown to be equivalent to the Frisch elasticity of leisure. One of the most widely used specifications for $g$ is the unit elasticity one: $g(1-h)=B \ln (\phi(1-h))$, which obviously implies that $(22)$ is satisfied. The unit elasticity case is particularly interesting since it can be obtained by inverting the expression $1-h=k \exp (-\rho e)$, where $\rho=\frac{1}{B}$ and $k=\frac{1}{\phi}$. These results might be quite useful for applied work. First, applied researchers typically choose utilities of consumption from the NIARA family and either use $h=\Gamma(e)=1-k \exp (-\rho e)$ with some convex effort cost function, or they use a linear function for $\Gamma$ and a constant elasticity function for leisure. Our results show that for these cases the first-order approach tends to be valid under hidden savings as well, hence the optimal allocations can be characterized in greater detail.

Second, empirical evidence suggests that these conditions are likely to be satisfied in the data. To our best knowledge, all estimations for $u$ reveal NIARA, for example Guiso et al. (2001) find decreasing and convex relative risk aversion. It is difficult to test directly the LCDF condition as effort is not observable. However, note that our model after the appropriate change in variables has similar structure as standard labor supply models where agents have concave utility functions over 
leisure and their labor supply decision affects their (expected) earnings linearly. ${ }^{20}$ In this sense, we can relate our condition $\eta^{l} \leq 1$ to empirical Frisch elasticities of labor supply. First of all notice that if $g(1-h)=\frac{(1-h)^{1-1 / \sigma}}{1-1 / \sigma}$, then the Frisch elasticity of leisure is $\sigma$ while the Frisch elasticity of labor supply is given by $\eta^{h}=\sigma \frac{1-h}{h}$. Note that, practically all estimates lead to $h \leq 0.5$ implying that $\eta^{h} \geq \eta^{l}$. This implies that the $\eta^{l} \leq 1$ requirement is stricter than requiring $\eta^{h} \leq 1$. Nevertheless, virtually all empirical estimates of labor supply elasticity find it between 0 and 0.5 for men Heckman and MaCurdy (1980) find higher estimates for women, but their estimates are still below one. One of the most recent structural estimations is done by Domeij and Floden (2006) who find values for the Frisch elasticity of labor supply between .3 and .56 .

\section{Discussion and Extensions}

We now explore a few important relaxations to the conditions stated in Theorem 1.

\subsection{CRRA utility}

A major step in the proof of Theorem 1 is provided by Lemma 2, which establishes a (log-)convexity property for utility differences $u\left(c_{i+1}+b\right)-u\left(c_{i}+b\right)$ for all NIARA utility functions and for all assets $b$. A suitable convexity assumption on the distribution function $F_{i}(e)$ then guarantees concavity of the agent's problem in $(e, b)$.

In many cases, the convexity property of $u\left(c_{i+1}+b\right)-u\left(c_{i}+b\right)$ stated in Lemma 2 can be strengthened, so that the convexity assumption on the distribution function can be relaxed accordingly. As an important example, consider CRRA utility: $u(c)=c^{1-\gamma} /(1-\gamma)$, where $\gamma>0$. For a moment, let us replace the difference $u\left(c_{i+1}+b\right)-u\left(c_{i}+b\right)$ by the agent's marginal utility $u^{\prime}(c)$ at some point $c$. Given CRRA utility, the marginal utility of consumption satisfies

$$
\frac{u^{\prime \prime \prime}(c) u^{\prime}(c)}{u^{\prime \prime 2}}=1+\frac{1}{\gamma}
$$

If the left-hand side is at least 1 , we obtain log-convexity of the marginal utility of consumption. Since the left-hand side is now strictly larger than 1, we have a somewhat stronger property. Similarly, we also obtain a somewhat stronger property for the terms $u\left(c_{i+1}+b\right)-u\left(c_{i}+b\right)$ than the result established by Lemma 2 .

As a consequence, we can relax the LCDF condition and obtain the following result.

\footnotetext{
${ }^{20}$ The two models are not isomorphic, though. This implies that we cannot derive $\eta^{l}$ as the Frisch elasticity of leisure in our environment.
} 
Proposition 3 Let $\tau$ be a transfer scheme that solves $(\mathbf{R})$. Suppose MLR and CRRA utility, $u(c)=$ $c^{1-\gamma} /(1-\gamma)$. If for all $e \geq 0, i=1, \ldots, N$, we have

$$
\frac{F_{i}^{\prime \prime}(e) F_{i}(e)}{F_{i}^{\prime 2}(e)} \geq \frac{\gamma}{1+\gamma}
$$

then, given $\tau$, the agent's decision problem is concave. Hence, $\tau$ solves the unrelaxed problem $(\mathbf{P})$.

Note that the right-hand side of (23) is a number between 0 and 1 . Thus, the convexity property imposed on the distribution function is somewhere between Rogerson's (1985b) CDF condition and the LCDF condition employed in Theorem 1.

\subsection{Quadratic Utility}

A quite commonly used specification for $u$ is the quadratic one: $u(c)=-\frac{\phi}{2}(B-c)^{2}$ with $\phi>0$ and $B>>0$. This utility function does not belong to the NIARA class since $-\frac{u^{\prime \prime}}{u^{\prime}}=\frac{1}{B-c}$, which increases with $c$. Most of the previous results however extend to this case.

The optimality conditions for problem $(\mathbf{R})$ when $u$ is quadratic are

$$
\frac{q-\xi \phi \beta}{\beta \phi\left(B-c_{i}\right)}=\lambda+\mu \frac{p_{i}^{\prime}\left(e_{0}\right)}{p_{i}\left(e_{0}\right)}
$$

It might hence be interesting to notice that, since $q-\xi \phi \beta>0,{ }^{21}$ the movements in the likelihood ratio are reflected in the optimal contract in the same way as in the standard moral hazard model. In particular, under MLR, consumption is monotone increasing in $i$ (as in the benchmark case with NIARA utility). Assuming the spanning condition, for simplicity, a sufficient condition for the validity of the first-order approach in this case is $f_{\Gamma}(e)(1-\Gamma(e)) \leq \frac{1+q}{2}$ for $e \geq 0$, which is implied by $f_{\Gamma}(e) \leq \frac{1+q}{2}$ since $1-\Gamma(e) \leq 1 .^{22}$

${ }^{21}$ If $q-\xi \phi \beta<0, c_{i}$ would decrease with $i$ when $\frac{p_{i}^{\prime}\left(e_{0}\right)}{p_{i}\left(e_{0}\right)}$ increases and vice versa. Hence:

$$
\sum p_{i}\left(e_{0}\right) \frac{p_{i}^{\prime}\left(e_{0}\right)}{p_{i}\left(e_{0}\right)} u\left(c_{i}\right)=\sum p_{i}^{\prime}\left(e_{0}\right) u\left(c_{i}\right)<0 \leq v^{\prime}\left(e_{0}\right)
$$

which violates the first-order incentive compatibility constraint for $e_{0}$.

${ }^{22}$ Assume without loss of generality that $v(e)=e$, and for simplicity that $\beta=q$. After a few simplifications, on the Hessian matrix

we obtain

$$
H:=\left[\begin{array}{ll}
-\beta \sum p_{i}^{\prime \prime}(e) \frac{\phi}{2}\left(B-c_{i}\right)^{2} & \beta \sum p_{i}^{\prime}(e) \phi\left(B-c_{i}\right) \\
\beta \sum p_{i}^{\prime}(e) \phi\left(B-c_{i}\right) & -\phi \beta[q+1]
\end{array}\right],
$$

$$
\operatorname{det} H=\beta^{2} \phi^{2}\left(\Gamma^{\prime \prime}(e)\left[\sum \Delta \pi_{i}\left(B-c_{i}\right)^{2}\right] \frac{(q+1)}{2}-\left(\Gamma^{\prime}(e)\right)^{2}\left(\sum \Delta \pi_{i}\left(B-c_{i}\right)\right)^{2}\right),
$$




\subsection{Exploiting the Curvature of the Contract}

To validate the first-order approach, we have previously derived conditions on the probability shifting functions and the agent's preferences under which, given monotonicity of the contract that solves (R), the agent's decision problem is concave. Finding such conditions will be much simpler if the contract that solves $(\mathbf{R})$ is not only monotonic, but also exhibits some form of concavity. For the moral hazard problem without hidden saving, Jewitt (1988) provides a general analysis of this idea. He shows that, under quite general conditions, the agent's (ex-post) utility changes with output in a concave way, and therefore the convexity conditions on the distribution function can be substantially relaxed.

In the present section, we try to exploit the curvature of the contract in the context of hidden saving. First of all, to avoid some complications associated with the discrete notion of concavity, we find it useful to formulate output as a continuous variable throughout this section. Hence, we replace the discrete output space $\left\{y_{1}, \ldots, y_{N}\right\}$ by the interval $Y:=[y, \bar{y}]$. Analogously, we replace the weights $p_{i}(e)$ by a probability density $f(y, e)$, with $f(y, e)>0$ for all $y, e$. Consumption schemes obtain the format $\mathbf{c}=\left(c_{0}, c(\cdot)\right)$, with $c_{0} \in[\underline{c}, \infty), c: Y \rightarrow[\underline{c}, \infty)$. For continuous output, the agent's preferences are specified as

$$
u\left(c_{0}-q b\right)-v(e)+\beta \int_{\underline{y}}^{\bar{y}} u(c(y)+b) f(y, e) d y,
$$

while the planner's objective function is

$$
\left(y_{0}-c_{0}\right)+q \int_{\underline{y}}^{\bar{y}}(y-c(y)) f(y, e) d y .
$$

The associated reformulations of the optimization problem $(\mathbf{P})$ and the relaxed problem $(\mathbf{R})$ are straightforward.

\subsubsection{Integration of the Distribution Function}

For the standard moral hazard problem, Jewitt (1988, Theorem 1) validates the first-order approach when the agent's (ex-post) consumption utility $u(c(y))$ is concave in output $y$ and the integral of the distribution function satisfies a convexity property. He then relates the latter property to the concept of Total Positivity (Karlin 1968) and shows that it is satisfied for a rather general class of probability distributions. In the setting with hidden assets, such a result is more difficult to obtain. Since the with $\Delta \pi_{i}:=\pi_{i h}-\pi_{i l}$, which implies that $\operatorname{det} H>0$ whenever

$$
\frac{\left[-\sum \Delta \pi_{i}\left(B-c_{i}\right)^{2}\right]}{\left(\sum \Delta \pi_{i}\left(B-c_{i}\right)\right)^{2}} \frac{(q+1)}{2}>\frac{\left(\Gamma^{\prime}(e)\right)^{2}}{-\Gamma^{\prime \prime}(e)} .
$$

Finally, one can show that - since $c_{i}$ and $\Delta \pi_{i}$ move together (and $\sum \Delta \pi_{i}=0$ ) - we have that $\frac{\sum \Delta \pi_{i}\left(B-c_{i}\right)^{2}}{\left(\sum \Delta \pi_{i}\left(B-c_{i}\right)\right)^{2}} \geq 1$. Q.E.D. 
agent's consumption level can be changed by saving, we need to specify not only the curvature between utility and output, but also how this curvature changes with the savings level $b$.

Proposition 4 Let $(\tau)$ be a transfer scheme that solves $(\mathbf{R})$. Suppose MLR and NIARA. Suppose that for all output levels $y$

$$
\begin{aligned}
& -\frac{d^{2}(u(c(y)+b))}{d y^{2}} \text { is positive and log-convex in } b, \\
& \widetilde{F}(y, e)=\int_{\underline{y}}^{y} F(z, e) d z \quad \text { is log-convex in } e .
\end{aligned}
$$

Then, given $(\tau)$, the agent's decision problem is concave. Hence, $(\tau)$ solves the unrelaxed problem $(\mathbf{P})$.

Condition (26) is a concavity property of the contract: The agent's consumption utility, $u(c(y)+b)$, depends on output $y$ in a concave way. In addition, the curvature between utility and output changes with assets $b$ in a log-convex way. It is not difficult to show that (26) is satisfied, for instance, if the consumption scheme $c(y)$ is concave in output $y$ and the agent has nonincreasing absolute prudence.

To capture the second condition in Proposition 4, it is important to note that log-convexity is preserved under integration (Boyd and Vandenberghe, p. 106). Therefore, log-convexity of the integral $\widetilde{F}(y, e)=\int_{\underline{y}}^{y} F(z, e) d z$ is a weaker assumption than log-convexity of the distribution function itself (LCDF). Intuitively, the integral $\widetilde{F}(y, e)$ will be log-convex in $e$ if the distribution function $F(y, e)$ is log-convex in $e$ for small values of $y$ and 'not too misbehaved' for large values of $y$.

One example that does not satisfy the LCDF property, but does satisfy (27), is the following.

Example 3 Consider the Beta Prime distribution with parameter $b=2$ :

$$
f(y, e)=\frac{y^{e-1}(1+y)^{-e-2}}{B(e, 2)}, \quad y \in[0, \infty), e \in[0, \infty),
$$

where $B(e, b)$ represents the Beta function. The likelihood ratio function $f_{e}(y, e) / f(y, e)$ is nondecreasing concave in $y$, hence the class of preferences satisfying (26) is nonempty. The distribution function is

$$
F(y, e)=(1+e+y) y^{e}(1+y)^{-e-1} .
$$

It is easy to see that $F(y, e)$ is not log-convex (not even convex) in $e$ for all $y$. However, the integral of the distribution function,

$$
\widetilde{F}(y, e)=y\left(\frac{y}{1+y}\right)^{e},
$$

is log-linear in $e$. Therefore, (27) is satisfied. 
However, the gain of Proposition 4 in terms of relaxing the LCDF condition is limited. It is difficult to find many other examples that satisfy (27) without satisfying LCDF. In addition, condition (26) is a stronger requirement than concavity of the agent's utility in output, since it also imposes structure on the link between the second derivative and the savings level. Given the nature of the problem, it seems impossible to avoid such a condition. ${ }^{23}$ Hence, compared to Jewitt's (1988) Theorem 1, we see that it is much more difficult to relax the convexity properties of the distribution function in the present framework.

\subsubsection{Quasiconvex Distribution Functions}

To exploit the curvature of contracts, another approach is to study distribution functions that are (jointly) quasiconvex in output and effort, because, roughly speaking, such distributions are equivalent to production functions with nonincreasing returns to scale. Jewitt (1988, Theorem 3) and Conlon (2009) use this property to validate the first-order approach for multi-signal moral hazard problems. In the present section, we show that quasiconvex distribution functions also have appealing properties for the model with hidden borrowing and lending.

Recall that, to establish concavity of the agent's decision problem, it is sufficient to consider the agent's utility at date $t=1$ (Remark 2). Moreover, note that the distribution function $F(y, e)$ is quasiconvex in $(y, e)$ if and only if output can be represented by a 'production function' $y=\varphi(e, z)$ that is nondecreasing concave in $e$ and nondecreasing in the stochastic state of nature $z$ (Jewitt 1988, Lemma 2). Using the production function, we can write the agent's date-1 utility as

$$
\int_{\underline{y}}^{\bar{y}} u(c(y)+b) f(y, e) d y=\mathbf{E}[u(c(\varphi(e, z))+b)]
$$

where $\mathbf{E}[\cdot]$ denotes expectations with respect to the state of nature $z$. Now, notice that concavity is preserved under summation and under nondecreasing concave transformations. Hence, since $u$ is nondecreasing concave, the agent's decision problem will be concave in $(e, b)$ if $c(\varphi(e, z))$ is concave in $e$ for all $z \cdot{ }^{24}$ This insight is key for the following result.

Proposition 5 Let $(\tau)$ be a transfer scheme that solves $(\mathbf{R})$. Suppose that the following conditions

\footnotetext{
${ }^{23}$ The idea of the proof used in Theorem 1 does not suffer from this problem. Note that monotonicity of the contract for one savings level is equivalent to monotonicity for all savings levels.

${ }^{24}$ At first glance, this insight seems to suggest that the validity of the first-order approach is not affected by the introduction of hidden saving. Notice, however, that the shape of the consumption scheme is crucially influenced by the agent's ability to save; see equation (10).
} 
hold:

$$
\begin{aligned}
& F(y, e) \text { is quasiconvex in }(y, e), \\
& f_{e}(y, e) / f(y, e) \text { is nondecreasing and concave in } y \text { for all } e, \\
& g(c ; \xi):=q /\left(\beta u^{\prime}(c)\right)-\xi a(c) \text { is increasing and convex in } c \text { for all } \xi \geq 0 .
\end{aligned}
$$

Then, given $(\tau)$, the agent's decision problem is concave. Hence, $(\tau)$ solves the unrelaxed problem $(\mathbf{P})$.

The function $g$ defined in (33) links the likelihood ratio function $f_{e}(y, e) / f(y, e)$ to the shape of the contract: As the continuous version of the optimality condition (10), we have

$$
\frac{q}{\beta u^{\prime}(c(y))}=\lambda+\mu \frac{f_{e}\left(y, e_{0}\right)}{f\left(y, e_{0}\right)}+\xi a(c(y)), \quad y \in[\underline{y}, \bar{y}],
$$

with $\xi \geq 0$. Hence, $c(y)$ is characterized by

$$
c(y)=g^{-1}\left(\lambda+\mu \frac{f_{e}\left(y, e_{0}\right)}{f\left(y, e_{0}\right)} ; \xi\right) .
$$

Thus, since $g^{-1}$ is nondecreasing concave under assumption (33), concave likelihood ratio functions will generate concave consumption schemes $c(y)$ in this case.

As an important application of Proposition 5, consider CARA utility: $u(c)=-\exp (-\alpha c) / \alpha$. Then we have $g(c ; \xi)=q \exp (\alpha c) / \beta-\xi \alpha$. Obviously, this function is increasing and convex in $c$ for all $\xi$. Therefore, Proposition 5 validates the first-order approach for CARA utility if the distribution function $F(y, e)$ is quasiconvex in $(y, e)$ and the likelihood ratio function $f_{e}(y, e) / f(y, e)$ is nondecreasing concave in $y .^{25}$

A second example to which (a minor modification of) Proposition 5 can be applied is quadratic utility: $u(c)=-\frac{\phi}{2}(B-c)^{2}$, with $\phi>0$ and $B>>0$. Here, we obtain $g(c ; \xi)=\frac{q-\xi \phi \beta}{\beta \phi(B-c)}$, with $q-\xi \phi \beta>0$ (see Section 4.2). Hence, $g(c ; \xi)$ is increasing and convex in $c$ when $\xi$ is set to its optimal value.

There are other examples, such as CRRA utility, for which the function $g$ is not convex, however. In that case, the concavity property of the likelihood ratio function formulated in (32) has to be strengthened to obtain a concave consumption scheme. Essentially, the difficulty in obtaining concave consumption schemes is driven by the coefficient of absolute risk aversion, which tends to make the right-hand side of the optimality condition (34) less concave in $y$.

\footnotetext{
${ }^{25}$ In the present paper, preferences over consumption and effort are additively separable. For CARA utility and multiplicatively separable preferences, by contrast, the validation of the first-order approach becomes much simpler, since the agent's effort choice will be independent of his wealth level.
} 


\section{Conclusions}

This paper studies the two-period version of the dynamic moral hazard model when agents can borrow and save on a risk-free bond market and their asset accumulation decisions are not observable. We provide sufficient conditions under which the first-order approach (FOA) is applicable in this environment. In comparison to the conditions which are required in static case with observable savings (MLR, $\mathrm{CDF}$ ), we need to impose some further concavity on the problem. First of all, non-increasing absolute risk aversion (NIARA) with respect to consumption is imposed. Second, the way effort affects the probability distribution has to be concave enough or the disutility of effort has to be convex enough. The strengthening of the convexity condition on the distribution function is done using the concept of log-convexity. We also show that, under somewhat more specific conditions, the log-convexity condition on the distribution is analogous to the Frisch elasticity of leisure being less than one. One nice property of this set of sufficient conditions is that these restrictions on preferences are validated by empirical research. Another attractive aspect is that most popular functional forms used in applied research will satisfy these conditions.

With the help of the first-order approach, we also characterize the optimal contract in this environment. We focus particularly on how consumption depends on output. Similarly to the standard case, under the assumptions needed for the validity of the FOA, optimal consumption is monotonic in output. It seems that NIARA is important - but not essential - for the result. In a related paper (Ábrahám, Koehne, and Pavoni, 2009), we also show that, when agent's utility belongs to the HARA class, the presence of hidden savings changes the curvature of the optimal contract making consumption to change in a 'more convex way' with output.

From a more general perspective, we believe that one of the important contributions of our paper is the application of log-convexity techniques. This idea gives optimization problems with multiplicatively separable objectives a tractable convex structure and seems to be useful for a much more general class of economic models.

In addition to these main results, we investigated a few further properties of the optimal contract that we do not report in full detail for brevity. For example, we show that some of the previous results about the value of information in the standard case also hold in our model. Assume that the planner receives an observable and verifiable signal $s \in S$, and suppose that the FOA is valid in both the original environment and the new environment with the signal $s$. Then there is a new

contract $\left\{c_{i s}\right\}_{i=0, N}^{s \in S}$ that strictly dominates $\left\{c_{i}\right\}_{i=0, N}$ if and only if $s$ is informative about $e_{0}$ in the sense of Holmström (1979). This result represents an interesting distinguishing feature of our model with respect to the self-insurance framework where further insurance possibilities are not available for the agent. In that case, consumption always moves with both $i$ and $s$ as long as $y_{i s}$ changes with 
them, regardless of the informational content.

Second, we investigated how effort and consumption insurance compare to the observable asset case. Assume again that the FOA is valid. Taking the first-order conditions with respect to $e_{0}$, we obtain

$$
q \sum_{i} p_{i}^{\prime}\left(e_{0}\right)\left(y_{i}-c_{i}\right)+\lambda U_{e}\left(e_{0}, 0 ; \tau\right)-\mu U_{e e}\left(e_{0}, 0 ; \tau\right)+\xi U_{e b}\left(e_{0}, 0 ; \tau\right)=0 .
$$

Clearly, $U_{e}\left(e_{0}, 0 ; \tau\right)=0$. Moreover, it is not difficult to see that whenever the agent's problem is concave in $e_{0}$ then we have $-\mu U_{e e}\left(e_{0}, 0 ; \tau\right)+\xi U_{e b}\left(e_{0}, 0 ; \tau\right)<0 .{ }^{26}$ This implies $q \sum_{i} p_{i}^{\prime}\left(e_{0}\right)\left(y_{i}-c_{i}\right)=$ $q \sum_{i} p_{i}^{\prime}\left(e_{0}\right)\left(y_{i}-c_{i}\right)+\lambda U_{e}\left(e_{0}, 0 ; \tau\right)>0$. If we consider $\lambda$ as an exogenous parameter defining the relative Pareto weight of the agent, this can be interpreted as an inefficiency result. Because of the informational problems, the planner implements an effort level which is lower than that dictated by production efficiency. This result is similar to the standard moral hazard case (see Rogerson, 1985b). A further natural question is whether the optimal scheme under hidden savings induces more or less consumption dispersion on the agent, given an effort level $e_{0}$. We realized that this question does not have an easy answer. Intuition might suggest that since reducing consumption dispersion relaxes the savings incentives under NIARA, we might expect a reduction of consumption dispersion. In fact, in Ábrahám and Pavoni (2006), we document numerically that in the two output case $(N=2)$ of the infinite horizon version of the model, consumption is typically more dispersed in the hidden asset case. A similar result can be shown analytically here: For each fixed pair $\left(U_{0}, e_{0}\right)$, the consumption dispersion is larger in our model than in the standard case. ${ }^{27}$ Given that the same effort level requires higher consumption dispersion, hence higher costs to the planner, we conjecture that - given $U_{0}$ hidden savings will induce a lower optimal level of effort compared to the standard case.

Finally, we would like to discuss the relevance of the assumption that neither the agent nor the principal are liquidity constrained in our model. Suppose that the agent is liquidity constrained. Although the set of implementable levels of asset holdings $b_{0}$ might be restricted by the presence of

\footnotetext{
${ }^{26}$ In particular, by rearranging the necessary conditions of optimality one can show the multipliers $\mu>0$ and $\xi>0$ are related as follows (details are available upon request): $\mu U_{e b}=\xi U_{b b}$. Hence, the condition $\operatorname{det} H>0$ is equivalent to

$$
\left(U_{e b}\right)^{2}<U_{e e} U_{b b} \Leftrightarrow \mu \xi U_{b b} U_{e b}<\mu^{2} U_{e e} U_{b b} \Leftrightarrow \xi U_{e b}<\mu U_{e e} .
$$

${ }^{27}$ Since $e_{0}$ is fixed across the two models, when $N=2$ we need to keep $\Delta u:=u\left(c_{H}\right)-u\left(c_{L}\right)$ constant across the two models. If $c_{0}$ were fixed as well, in order to satisfy the Euler equation in our model, we need to decrease $p\left(e_{0}\right) u^{\prime}\left(c_{H}\right)+\left(1-p\left(e_{0}\right)\right) u^{\prime}\left(c_{L}\right)$ compared to the standard moral hazard model. The implication is that we must increase both $c_{H}$ and $c_{L}$ so that to keep $\Delta u$ constant. Since $u$ is concave, $\Delta c$ must increase. Now, allow for the possibility of changing $c_{0}$. As long as both $U_{0}$ and $e_{0}$ are fixed, the principal perhaps decreases $c_{0}$, but again in order to keep $\Delta u$ constant, both $c_{L}$ and $c_{H}$ must increase compared to the original contract, otherwise the agent's participation constraint would be violated.
} 
liquidity constraints, all our characterization results remain valid. First, it is obvious that given any implementable $b_{0}$, the planner is able to generate the same allocation to the agent by implementing $b_{0}^{\prime}>b_{0}$ and adjusting transfers accordingly at no cost. Interestingly, Lemma 1 implies that whenever the FOA is valid the planner will never be able to gain by implementing a low $b_{0}$ so that to make the agent liquidity constrained. In contrast, when both the agent and the planner faces liquidity constraints (or shallow pockets), it might be the case that Lemma 1 fails as $U_{b}>0$.

\section{References}

[1] Ábrahám, Á. and N. Pavoni (2006) "Efficient Allocations with Moral Hazard and Hidden Borrowing and Lending," mimeo, http://www.ucl.ac.uk/ uctpnpa/abpav.pdf .

[2] Ábrahám, Á. and N. Pavoni (2008), "Efficient Allocations with Moral Hazard and Hidden Borrowing and Lending: A Recursive Formulation," Review of Economic Dynamics, 11(4): 781-803.

[3] Ábrahám, Á., S. Koehne, and N. Pavoni (2009) "Optimal Income Taxation and Hidden Savings," mimeo, University College London.

[4] Allen, F. (1985), "Repeated Principal-Agent Relationship with Lending and Borrowing," Economic Letters, 17: 27-31.

[5] Atkeson, A. (1991), "International Lending with Moral Hazard and Risk of Repudiation," Econometrica 59(4), July: 1069-1089.

[6] Bertola, G., and W. Koeninger (2009), "Public and Private Insurance," mimeo, Queen Mary University, and Collegio Carlo Alberto.

[7] Bisin, A., and A. Rampini (2006) "Exclusive Contracts and the Institution of Bankruptcy," Economic Theory, 27, 277-304.

[8] Bizer, D. S. and P. M. DeMarzo (1999), "Optimal Incentive Contracts When Agents can Save, Borrow and Default" Journal of Financial Intermediation, 8: 241-269.

[9] Boyd, S., and L. Vandenberghe (2004), Convex Optimization, Cambridge University Press.

[10] Chade, H. (2009), "Moral Hazard with Unobservable Consumption," mimeo, Arizona State University.

[11] Chiappori, P. A., I. Macho, P. Rey and B. Salanié (1994), "Repeated Moral Hazard: The Role of Memory, Commitment, and Access to Credit Markets," European Economic Review, 1527-1553. 
[12] Cole, H. L. and N. R. Kocherlakota (2001), "Efficient Allocations with Hidden Income and Hidden Storage," Review of Economic Studies, 68(3): 523-542.

[13] Conlon, J. R. (2009), "Two New Conditions Supporting the First-Order Approach to Multisignal Principal-Agent Problems," Econometrica, 77(1): 249-278.

[14] Domeij, D. and M. Floden (2006), "The Labor-supply elasticity and borrowing constraints: Why estimates are biased," Review of Economic Dynamics, 9: 242-262.

[15] Gollier, C. (2001) The Economics of Risk and Time, MIT Press.

[16] Gottardi, P. and N. Pavoni, (2008), "Ramsey Asset Taxation Under Asymmetric Information," mimeo, University of Venice and UCL.

[17] Grossman, S. and O. Hart, (1983), "An Analysis of the Principal-Agent Problem," Econometrica, 51: 7-45.

[18] Guiso, L. and M. Paiella, (2001), "Risk Aversion, Wealth and Background Risk," CEPR DP 2728.

[19] Heckman, J. and T. MaCurdy (1980). "A Dynamic Model of Female Labor Supply," Review of Economic Studies. 47(1): 47-74.

[20] Holmström, B. (1979). "Moral Hazard and Observability." Bell Journal of Economics 10:74-91.

[21] Hopenhayn, H. and J. P. Nicolini (1997), "Optimal Unemployment Insurance," Journal of Political Economy, 105(2): 412-438.

[22] Jewitt, I. (1988), "Justifying the First-Order Approach to Principal-Agent Problems," Econometrica, 56(5): 1177-1190.

[23] Karlin, S. (1968), Total Positivity: Volume 1, Stanford University Press, Stanford CA.

[24] Kocherlakota, N. (2004), "Figuring out the Impact of Hidden Savings on Optimal Unemployment Insurance," Review of Economic Dynamics: 7(3) July 2004: 541-554.

[25] Kocherlakota, N. (2005), "Zero Expected Wealth Taxes: A Mirrlees Approach to Dynamic Optimal Taxation," Econometrica, 74.

[26] Mirrlees, J. (1974), "Notes on Welfare Economics, Information and Uncertainty", in Essays in Economic Behavior Under Uncertainty, ed. by M. Balch, D. McFadden, and S. Wu. NorthHolland, Amsterdam. 
[27] Mirrlees, J. (1975), "The Theory of Moral Hazard and Unobservable Behaviour: Part I", mimeo, Nuffield College, Oxford. Reprinted in the Review of Economic Studies, (1999), 66: 3-21.

[28] Mirrlees, J. (1979), "The Implications of Moral Hazard for Optimal Insurance," mimeo, Seminar given at Conference held in honor of Karl Borch, Bergen, Norway.

[29] Mitchell, M., and Y. Zhang (2009), "Unemployment Insurance with Hidden Savings," mimeo, University of Toronto.

[30] Park In-Uck (2004), "Moral Hazard Contracting and Private Credit Markets," Econometrica, 72(3), May: 701-746.

[31] Raith, M. (2008), "Optimal Incentives and the Time Dimension of Performance Measurement," mimeo, University of Rochester.

[32] Rogerson, W. (1985a), "Repeated Moral Hazard," Econometrica, 53: 69-76.

[33] Rogerson, W. (1985b), "The First-Order Approach to Principal-Agent Problems," Econometrica, 53: $1357-1368$.

[34] Williams, N. (2006), "On Dynamic Principal-Agent Problems in Continuous Time," mimeo, Princeton University.

[35] Werning, I. (2001), "Repeated Moral-Hazard with Unmonitored Wealth: A Recursive First-Order Approach," mimeo, MIT.

[36] Werning, I. (2002), "Optimal Unemployment Insurance with Unobservable Savings," mimeo, MIT. 


\section{Appendix: Proofs}

Proof of Proposition 1 The proof is based on Propositions 4 and 5 in Grossman and Hart (1983). In order to make the analogy explicit between these results and ours, we now show in detail the part of the proof related to Grossman and Hart's Proposition 5 which regards our Corollary to Proposition 1: The sub-optimality of self-insurance. It should then be easy to see how the proof of Proposition 4 in that paper can be adapted to our environment. Further details are available upon request.

Consider a transfer scheme $\tau$ that is optimal and it is such that $\tau_{i} \equiv \tau$ is constant for all $i>$ 0 . We show that this transfer scheme cannot be optimal, by constructing an incentive compatible transfer scheme, which satisfies the agent's participation constraint and increases the planner's surplus. Without loss of generality, assume that $u$ is unbounded below and recall that $\tau_{N}=\tau_{1}$. Consider the following modification of the scheme: leave unchanged both $\hat{\tau}_{0}(\varepsilon)=\tau_{0}$ and $\hat{\tau}_{i}(\varepsilon)=\tau$ for $i=2, \ldots, N-1$, while modify $\tau_{1}$ and $\tau_{N}$ as follows: set $\hat{\tau}_{1}(\varepsilon)=\tau+\varepsilon$, and $\hat{\tau}_{N}(\varepsilon)=\tau-\mu^{\varepsilon} \varepsilon$, with $\varepsilon>0$. For any $\varepsilon$, the value $\mu^{\varepsilon}$ is chosen so that the agent is indifferent between the original plan $\tau$ and the new one $\hat{\tau}(\varepsilon)^{28}$, that is

$$
\begin{aligned}
& \max _{e \in E, b \geq-B(\varepsilon)} u\left(y_{0}+\tau_{0}-q b\right)-v(e)+\beta \sum_{i=1}^{N} p_{i}(e) u\left(y_{i}+\hat{\tau}_{i}(\varepsilon)+b\right) \\
= & \max _{e \in E, b \geq-B(\varepsilon)} u\left(y_{0}+\tau_{0}-q b\right)-v(e)+\beta \sum_{i=2}^{N-1} p_{i}(e) u\left(y_{i}+\tau+b\right) \\
& +\beta\left[p_{1}(e) u\left(y_{1}+\tau+\varepsilon+b\right)+p_{N}(e) u\left(y_{N}+\tau-\mu^{\varepsilon} \varepsilon+b\right)\right] \\
= & u\left(y_{0}+\tau_{0}\right)-v\left(e_{0}\right)+\beta \sum_{i} p_{i}\left(e_{0}\right) u\left(y_{i}+\tau\right) .
\end{aligned}
$$

where for each $\varepsilon, B(\varepsilon):=\min _{i}\left\{y_{i}+\hat{\tau}_{i}(\varepsilon)-\underline{c}\right\}$, and we stick to the particular optimal contract where $b_{0}=0$. Such a $\mu^{\varepsilon}>0$ exists by the Maximum Theorem. Notice indeed that both $u$ and $\mathbf{p}$ are continuous and by convexity and strict monotonicity, $e$ will lie in a compact interval. Moreover, since the transfers are constant in $i$, and utility is unbounded below we can always choose $\varepsilon$ in a way that $b_{0}(\varepsilon) \in B(\varepsilon, \mu)$ where $B(\varepsilon, \mu):=\left[\underline{c}-\min \left\{y_{1}+\tau+\varepsilon, y_{N}+\tau-\mu \varepsilon\right\}, \frac{y_{0}+\tau_{0}-\underline{c}}{q}\right]$ is a non-empty and compact set (recall that $\underline{c}>-\infty$ ). Finally, note that for each $\varepsilon>0$, for $\mu=0$ the utility of the agent must be strictly larger than the one in the original contract. Since $u$ is strictly monotone and unbounded below, and by the full support assumption, by reducing $\mu$ we can drive the agent's utility arbitrarily low. Hence, by continuity, there must be a $\mu^{\varepsilon}$ that satisfies our requirement.

We want to show that for $\varepsilon$ small enough the difference between the planner's surplus under the new and original scheme $\delta(\varepsilon)$ is positive. Denote by $\hat{e}_{0}^{\varepsilon}$ and $\hat{b}_{0}^{\varepsilon}$ the effort and asset choices of the agent

\footnotetext{
${ }^{28}$ When $u$ is unbounded above, we shall modify the original scheme $\tau$ as follows: $\hat{\tau}_{0}(\varepsilon)=\tau_{0}, \hat{\tau}_{i}(\varepsilon)=\tau$ for $i=$ $2, \ldots, N-1$, and $\hat{\tau}_{1}(\varepsilon)=\tau+\mu^{\varepsilon} \varepsilon$, and $\hat{\tau}_{N}(\varepsilon)=\tau-\varepsilon$.
} 
under the perturbed scheme, and $\hat{e}_{0}^{0}, \hat{b}_{0}^{0}$ and $\mu^{0}$ are the limit effort, asset and $\mu^{\varepsilon}$ choices as $\varepsilon \rightarrow 0^{+}$. The Theorem of the Maximum implies that the optimal correspondence of choices $\hat{e}_{0}^{\varepsilon}$ and $\hat{b}_{0}^{\varepsilon}$ is upper hemi-continuous if considered as a function of $\varepsilon$. The consequence is that $\hat{e}_{0}^{0}$ and $\hat{b}_{0}^{0}$ are also optimal under the original scheme, hence for each $\varepsilon>0$ we have

$$
\begin{aligned}
& u\left(y_{0}+\tau_{0}-q \hat{b}_{0}^{0}\right)-v\left(\hat{e}_{0}^{0}\right)+\beta \sum_{i} p_{i}\left(\hat{e}_{0}^{0}\right) u\left(y_{i}+\hat{\tau}_{i}(\varepsilon)+\hat{b}_{0}^{0}\right) \\
\leq & u\left(y_{0}+\tau_{0}-q \hat{b}_{0}^{\varepsilon}\right)-v\left(\hat{e}_{0}^{\varepsilon}\right)+\beta \sum_{i} p_{i}\left(\hat{e}_{0}^{\varepsilon}\right) u\left(y_{i}+\hat{\tau}_{i}(\varepsilon)+\hat{b}_{0}^{\varepsilon}\right) \\
= & u\left(y_{0}+\tau_{0}-q \hat{b}_{0}^{0}\right)-v\left(\hat{e}_{0}^{0}\right)+\beta \sum_{i} p_{i}\left(\hat{e}_{0}^{0}\right) u\left(y_{i}+\tau+\hat{b}_{0}^{0}\right) .
\end{aligned}
$$

Comparing the first line with the last one, we have:

$$
\sum_{i} p_{i}\left(\hat{e}_{0}^{0}\right)\left[u\left(y_{i}+\hat{\tau}_{i}(\varepsilon)+\hat{b}_{0}^{0}\right)-u\left(y_{i}+\tau+\hat{b}_{0}^{0}\right)\right] \leq 0 .
$$

Since $\varepsilon, \mu^{\varepsilon}>0$, condition (35) can be rewritten as

$$
\begin{aligned}
& \varepsilon p_{1}\left(\hat{e}_{0}^{0}\right) \frac{\left[u\left(y_{1}+\tau+\varepsilon+\hat{b}_{0}^{0}\right)-u\left(y_{1}+\tau+\hat{b}_{0}^{0}\right)\right]}{\varepsilon} \\
& -\mu^{\varepsilon} \varepsilon p_{N}\left(\hat{e}_{0}^{0}\right) \frac{\left[u\left(y_{N}+\tau+\hat{b}_{0}^{0}\right)-u\left(y_{N}+\tau-\mu^{\varepsilon} \varepsilon+\hat{b}_{0}^{0}\right)\right]}{\mu^{\varepsilon} \varepsilon} \leq 0 .
\end{aligned}
$$

Now taking limits as $\varepsilon \rightarrow 0^{+}$we get

$$
0 \geq p_{1}\left(\hat{e}_{0}^{0}\right) u^{\prime}\left(y_{1}+\tau+\hat{b}_{0}^{0}\right)-p_{N}\left(\hat{e}_{0}^{0}\right) \mu^{0} u^{\prime}\left(y_{N}+\tau+\hat{b}_{0}^{0}\right) .
$$

It is now easy to realize that $y_{N}+\tau>y_{1}+\tau$ and strict concavity implies $u^{\prime}\left(y_{1}+\tau+\hat{b}_{0}^{0}\right)>$ $u^{\prime}\left(y_{N}+\tau+\hat{b}_{0}^{0}\right)$ hence it must be that $p_{N}\left(\hat{e}_{0}^{0}\right) \mu^{0}-p_{1}\left(\hat{e}_{0}^{0}\right)>0$. Let us now compute the gain for the planner. For each $\varepsilon>0$ we have

$$
\delta(\varepsilon)=\sum_{i} p_{i}\left(e_{0}\right) \tau-\sum_{i} p_{i}\left(e_{0}^{\varepsilon}\right) \hat{\tau}_{i}(\varepsilon)=p_{N}\left(\hat{e}_{0}^{\varepsilon}\right) \mu^{\varepsilon} \varepsilon-p_{1}\left(\hat{e}_{0}^{\varepsilon}\right) \varepsilon .
$$

Since $\delta(0)=0$, by showing $\delta^{\prime+}(0)>0$, we show that $\delta(\varepsilon)>0$ for $\varepsilon$ small enough. Note that if we divide (38) by $\varepsilon$ and we take the limit as $\varepsilon \rightarrow 0^{+}$we get

$$
\delta^{\prime+}(0)=\lim _{\varepsilon \rightarrow 0^{+}} \frac{\delta(\varepsilon)}{\varepsilon}=p_{N}\left(\hat{e}_{0}^{0}\right) \mu^{0}-p_{1}\left(\hat{e}_{0}^{0}\right)>0 .
$$

\section{Q.E.D.}


Proof of Lemma 1 (i) We first show that the solution of the problem $(\mathbf{R})$ must be such that (7) (the no-shirking condition) is satisfied with equality. If $\mu>0$ we are done. Consider the case where $\mu=0$. In this case, the first-order conditions are either $c_{i}=\underline{c}$ for all $i$, or $\frac{q}{\beta u^{\prime}\left(c_{i}\right)}-\xi a\left(c_{i}\right)=\lambda$ for all $i$, hence the planner fully insures the agent in period $t=1$ regardless of the value of $\xi \geq 0$. Note that full-insurance will also be good for incentive compatibility since the convexity of $u^{\prime}$ (implied by NIARA) implies $\sum_{i} p_{i}\left(e_{0}\right) u^{\prime}\left(c_{i}\right) \geq u^{\prime}\left(\sum_{i} p_{i}\left(e_{0}\right) c_{i}\right)$. That is, the planner will be able to relax the Euler equation by providing insurance. Since $\sum_{i} p_{i}^{\prime}\left(e_{0}\right)=0$, a constant $u\left(c_{i}\right)=\bar{u}$ implies that $\sum_{i} p_{i}^{\prime}\left(e_{0}\right) \bar{u}=0$. Since $v^{\prime}\left(e_{0}\right) \geq 0$, full insurance would imply $v^{\prime}\left(e_{0}\right) \geq \beta \sum_{i} p_{i}^{\prime}\left(e_{0}\right) u\left(c_{i}\right)=0$. Combining this with (7) delivers $v^{\prime}\left(e_{0}\right)=\beta \sum_{i} p_{i}^{\prime}\left(e_{0}\right) u\left(c_{i}\right)=0$.

We now show that if the solution of the problem $(\mathbf{R})$ is interior for $c_{i}$, it must be such that the second constraint in (6) (the Euler condition) is satisfied with equality. Recall that the first-order conditions for $c_{0}$ and $c_{i}$ are given by (9) and (10). Again, if $\xi>0$ we are done. If $\xi=0$, from the first order conditions we have:

$$
\frac{1}{u^{\prime}\left(c_{0}\right)} \geq \sum_{i} p_{i}\left(e_{0}\right) \frac{q}{\beta u^{\prime}\left(c_{i}\right)} \geq \frac{q}{\beta \sum_{i} p_{i}\left(e_{0}\right) u^{\prime}\left(c_{i}\right)}
$$

where the second inequality is implied by Jensen inequality. In fact, since $1 / x$ is a strictly convex transformation, this second inequality can be an equality only if the agent is fully insured. Now, comparing the first and last term in the above expression, we have $q u^{\prime}\left(c_{0}\right) \leq \beta \sum_{i} p_{i}\left(e_{0}\right) u^{\prime}\left(c_{i}\right)$. Combining this with (8) yields $q u^{\prime}\left(c_{0}\right)=\beta \sum_{i} p_{i}\left(e_{0}\right) u^{\prime}\left(c_{i}\right)$.

(ii) Whenever $e_{0}>0$ by assumption we have $v^{\prime}\left(e_{0}\right)>0$. Therefore, full insurance is not feasible so $c_{i}$ cannot be constant in $i$. This implies that $\mu>0$. In this case we also have $\xi>0$. In order to see this, recall that with $\xi=0$ whenever $c_{i}$ is not constant in $i$ the last inequality in (39) is satisfied with strict inequality. This however contradicts the Euler condition $q u^{\prime}\left(c_{0}\right) \geq \beta \sum_{i} p_{i}\left(e_{0}\right) u^{\prime}\left(c_{i}\right)$ hence $\xi$ cannot be zero either.

(iii) If $u$ is unbounded below, since $U_{0}>-\infty$, from the participation constraint we obviously have interiority and the previous line of proof applies. So assume $u$ is bounded. If the Inada condition is satisfied and $c_{i}=\underline{c}$ for at least one $i>0$, clearly the Euler condition (8) must be satisfied. If fact, it must be that $c_{0}=\underline{c}$ as well. Since $u$ is bounded, (7) must be satisfied with equality by the argument in (i). Q.E.D.

Proof of Lemma 2 Recall that a twice differentiable function $f: I \rightarrow \mathbb{R}_{++}$, where $I$ is a real interval, is log-convex if and only if $f^{\prime \prime} f / f^{\prime 2} \geq 1$. Therefore, setting $\hat{c}_{i}:=c_{i}+b$, we note that $u\left(c_{i+1}+b\right)-u\left(c_{i}+b\right)$ is log-convex in $b$ if and only if

$$
\frac{\left(u\left(\hat{c}_{i+1}\right)-u\left(\hat{c}_{i}\right)\right)\left(u^{\prime \prime}\left(\hat{c}_{i+1}\right)-u^{\prime \prime}\left(\hat{c}_{i}\right)\right)}{\left(u^{\prime}\left(\hat{c}_{i+1}\right)-u^{\prime}\left(\hat{c}_{i}\right)\right)^{2}} \geq 1 .
$$


First, suppose that $u$ exhibits the NIARA property. Then the agent is more prudent than risk averse, thus $-u^{\prime}$ is a concave transformation of $u$ (Gollier 2001, p. 52). In other words, there is a nondecreasing concave function $f$ such that

$$
-u^{\prime}(c)=f(u(c)) \text { for all } c .
$$

Now, note that

$$
u^{\prime \prime}(c)=\frac{d u^{\prime}(c)}{d c}=-\frac{d\left(-u^{\prime}(c)\right.}{d c}=-f^{\prime}(u(c)) u^{\prime}(c)=f^{\prime}(u(c)) f(u(c)) .
$$

Using this result, and setting $u\left(\hat{c}_{i}\right)=u_{i}$, we obtain

$$
\frac{\left(u\left(\hat{c}_{i+1}\right)-u\left(\hat{c}_{i}\right)\right)\left(u^{\prime \prime}\left(\hat{c}_{i+1}\right)-u^{\prime \prime}\left(\hat{c}_{i}\right)\right)}{\left(u^{\prime}\left(\hat{c}_{i+1}\right)-u^{\prime}\left(\hat{c}_{i}\right)\right)^{2}}=\frac{\left(u_{i+1}-u_{i}\right)\left(f^{\prime}\left(u_{i+1}\right) f\left(u_{i+1}\right)-f^{\prime}\left(u_{i}\right) f\left(u_{i}\right)\right)}{\left(f\left(u_{i+1}\right)-f\left(u_{i}\right)\right)\left(f\left(u_{i+1}\right)-f\left(u_{i}\right)\right)} .
$$

By the mean value theorem, there exists a real number $\tilde{u} \in\left[u_{i}, u_{i+1}\right]$ such that

$$
f\left(u_{i+1}\right)-f\left(u_{i}\right)=f^{\prime}(\tilde{u})\left(u_{i+1}-u_{i}\right)
$$

Therefore, (41) can be rewritten as

$$
\frac{f\left(u_{i+1}\right) f^{\prime}\left(u_{i+1}\right) / f^{\prime}(\tilde{u})-f\left(u_{i}\right) f^{\prime}\left(u_{i}\right) / f^{\prime}(\tilde{u})}{f\left(u_{i+1}\right)-f\left(u_{i}\right)} .
$$

Since $f^{\prime}$ is nonincreasing, we have $f^{\prime}\left(u_{i}\right) \leq f^{\prime}(\tilde{u}) \leq f^{\prime}\left(u_{i+1}\right)$, which implies $f^{\prime}\left(u_{i+1}\right) / f^{\prime}(\tilde{u}) \leq 1 \leq$ $f^{\prime}\left(u_{i}\right) / f^{\prime}(\tilde{u})$. This, together with the fact that $f$ is negative, leads to

$$
\frac{f\left(u_{i+1}\right) f^{\prime}\left(u_{i+1}\right) / f^{\prime}(\tilde{u})-f\left(u_{i}\right) f^{\prime}\left(u_{i}\right) / f^{\prime}(\tilde{u})}{f\left(u_{i+1}\right)-f\left(u_{i}\right)} \geq 1
$$

or equivalently

$$
\frac{\left(u\left(\hat{c}_{i+1}\right)-u\left(\hat{c}_{i}\right)\right)\left(u^{\prime \prime}\left(\hat{c}_{i+1}\right)-u^{\prime \prime}\left(\hat{c}_{i}\right)\right)}{\left(u^{\prime}\left(\hat{c}_{i+1}\right)-u^{\prime}\left(\hat{c}_{i}\right)\right)^{2}} \geq 1 .
$$

Hence, $u\left(c_{i+1}+b\right)-u\left(c_{i}+b\right)$ is log-convex in $b$.

On the other hand, suppose that $u\left(c_{i+1}+b\right)-u\left(c_{i}+b\right)$ is log-convex in $b$ for all $c_{i+1}>c_{i}$. Then, dividing (40) by $\hat{c}_{i+1}-\hat{c}_{i}$ and taking limits, we obtain the characterizing condition for NIARA. Q.E.D.

Proof of Proposition 3 Let $u(c)=c^{1-\gamma} /(1-\gamma)$ for $c>0$. First, we want to establish the inequality

$$
\frac{\left(u\left(c_{i+1}+b\right)-u\left(c_{i}+b\right)\right)\left(u^{\prime \prime}\left(c_{i+1}+b\right)-u^{\prime \prime}\left(c_{i}+b\right)\right)}{\left(u^{\prime}\left(c_{i+1}+b\right)-u^{\prime}\left(c_{i}+b\right)\right)^{2}} \geq 1+\frac{1}{\gamma}
$$

for all $c_{i+1}+b>c_{i}+b>0$. 
To simplify notation, set $\hat{c}_{i}=c_{i}+b$. Note $u^{\prime}(c)=c^{-\gamma}, u^{\prime \prime}(c)=-\gamma c^{-\gamma-1}$. Therefore, (42) is equivalent to

$$
-\frac{\gamma\left(\hat{c}_{i+1}^{1-\gamma}-\hat{c}_{i}^{1-\gamma}\right)\left(\hat{c}_{i+1}^{-1-\gamma}-\hat{c}_{i}^{-1-\gamma}\right)}{(1-\gamma)\left(\hat{c}_{i+1}^{-\gamma}-\hat{c}_{i}^{-\gamma}\right)^{2}} \geq \frac{1+\gamma}{\gamma}
$$

which can be rewritten as

$$
\frac{-\gamma^{2}\left(1-\left(\hat{c}_{i} / \hat{c}_{i+1}\right)^{1-\gamma}\right)\left(1-\left(\hat{c}_{i} / \hat{c}_{i+1}\right)^{-1-\gamma}\right)}{(1-\gamma)(1+\gamma)\left(1-\left(\hat{c}_{i} / \hat{c}_{i+1}\right)^{-\gamma}\right)^{2}} \geq 1 .
$$

Hence, since $0 \leq \hat{c}_{i} / \hat{c}_{i+1} \leq 1$, it is sufficient to show

$$
-\frac{\gamma\left(1-x^{1-\gamma}\right)}{(1-\gamma)\left(1-x^{-\gamma}\right)} \cdot \frac{\gamma\left(1-x^{-1-\gamma}\right)}{(1+\gamma)\left(1-x^{-\gamma}\right)} \geq 1 \text { for all } x \in[0,1] .
$$

By l'Hospital's rule, we obtain

$$
\begin{aligned}
& \lim _{x \rightarrow 1} \frac{1-x^{1-\gamma}}{1-x^{-\gamma}}=-\frac{1-\gamma}{\gamma}, \\
& \lim _{x \rightarrow 1} \frac{1-x^{-1-\gamma}}{1-x^{-\gamma}}=\frac{1+\gamma}{\gamma} .
\end{aligned}
$$

Hence, the left-hand side of (43) converges to 1 as $x$ goes to 1 . Since the left-hand side of (43) is decreasing in $x$ for $x \leq 1$, this establishes (43) and hence (42).

Next, we claim that the convexity property of the distribution function expressed in (23), together with inequality (42), implies that the product

$$
F_{i}(e)\left[u\left(c_{i+1}+b\right)-u\left(c_{i}+b\right)\right]
$$

is convex in $(e, b)$. The Hessian of this product is given by

$$
H=\left[\begin{array}{ll}
F_{i}^{\prime \prime}(e)\left[u\left(c_{i+1}+b\right)-u\left(c_{i}+b\right)\right] & F_{i}^{\prime}(e)\left[u^{\prime}\left(c_{i+1}+b\right)-u^{\prime}\left(c_{i}+b\right)\right] \\
F_{i}^{\prime}(e)\left[u^{\prime}\left(c_{i+1}+b\right)-u^{\prime}\left(c_{i}+b\right)\right] & F_{i}(e)\left[u^{\prime \prime}\left(c_{i+1}+b\right)-u^{\prime \prime}\left(c_{i}+b\right)\right]
\end{array}\right] .
$$

Note that $F_{i}(e)$ and $u\left(c_{i+1}+b\right)-u\left(c_{i}+b\right)$ are both nonnegative. Hence, the first diagonal entry of $H$ is nonnegative by (23), the second one is nonnegative by (42). By combining (23) and (42) we see that also the determinant of $H$ is nonnegative. Hence, $H$ is positive semidefinite, which implies the convexity of $F_{i}(e)\left[u\left(c_{i+1}+b\right)-u\left(c_{i}+b\right)\right]$ in $(e, b)$. Now, the concavity of the agent's problem follows from the same steps as in Theorem 1. Q.E.D. 
Proof of Proposition 4 By Remark 2, it is sufficient to establish concavity of

$$
(e, b) \mapsto \int_{\underline{y}}^{\bar{y}} u(c(y)+b) f(y, e) d y .
$$

This is equivalent to establishing convexity of

$$
(e, b) \mapsto-\int_{\underline{y}}^{\bar{y}} u(c(y)+b) f(y, e) d y .
$$

Using two steps of partial integration, the latter function can be rewritten as

$$
\left.-u(c(\bar{y})+b)+c^{\prime}(\bar{y}) u^{\prime}(c(\bar{y})+b)\right) \widetilde{F}(\bar{y}, e)+\int_{\underline{y}}^{\bar{y}}\left(-\frac{d^{2}(u(c(y)+b))}{d y^{2}}\right) \widetilde{F}(y, e) d y .
$$

First, note that the expression $-u(c(\bar{y})+b)$ is convex in $(e, b)$ due to the concavity of $u$. Moreover, the expression

$$
\left.c^{\prime}(\bar{y}) u^{\prime}(c(\bar{y})+b)\right) \widetilde{F}(\bar{y}, e)
$$

is log-convex (hence convex) in $(e, b)$, since $u^{\prime}(c(\bar{y})+b)$ ) is log-convex in $b$ for NIARA utility, $\widetilde{F}(\bar{y}, e)$ is log-convex in $e$ by assumption, and since log-convexity is preserved under multiplication. For the third term in (44), note that

$$
-\frac{d^{2}(u(c(y)+b))}{d y^{2}} \widetilde{F}(y, e)
$$

is, by assumption, again a product of function that is log-convex in $b$ and a function that is log-convex in $e$. By the same argument as above, this product is convex in $(e, b)$. Since convexity is preserved under integration, the third term in (44) is thus convex as well. This completes the proof. Q.E.D.

Proof of Proposition 5 By Remark 2, it is sufficient to consider the agent's utility at date $t=1$. Moreover, due to quasiconvexity of the distribution function, the output technology can be represented by a production function $y=\varphi(e, z)$, with $\varphi(e, z)$ nondecreasing concave in effort $e$ (Jewitt 1988, Lemma 2). Using this representation, we can write the agent's date-1 utility as

$$
\int_{\underline{y}}^{\bar{y}} u(c(y)+b) f(y, e) d y=\mathbf{E}[u(c(\varphi(e, z))+b)]
$$

where $\mathbf{E}[\cdot]$ denotes expectations with respect to the state of nature $z$.

We want to show that the consumption scheme $c$ is nondecreasing concave. As continuous version of the optimality condition (10), we have

$$
\frac{q}{\beta u^{\prime}(c(y))}=\lambda+\mu \frac{f_{e}\left(y, e_{0}\right)}{f\left(y, e_{0}\right)}+\xi a(c(y)), \quad y \in[\underline{y}, \bar{y}] .
$$

Hence, $c$ is characterized by

$$
c(y)=g^{-1}\left(\lambda+\mu \frac{f_{e}\left(y, e_{0}\right)}{f\left(y, e_{0}\right)} ; \xi\right)
$$


with $g(c ; \xi)=q /\left(\beta u^{\prime}(c)\right)-\xi a(c)$. By assumption, $g$ is increasing and convex. Equivalently, $g^{-1}$ is increasing and concave. Since $f_{e}\left(y, e_{0}\right) / f\left(y, e_{0}\right)$ is nondecreasing concave in $y$ by assumption, this implies that $c(y)$ is nondecreasing concave in $y$.

Now, since $\varphi(e, z)$ is concave in $e$ and $c(y)$ is nondecreasing concave in $y$, the composition $c(\varphi(e, z))$ is concave in $e$. Hence, the function $c(\varphi(e, z))+b$ is concave in $(e, b)$. Since $u$ is nondecreasing concave, and since concavity is preserved under taking expectations, this completes the proof. Q.E.D. 